\title{
BUILDING UPON WEAKNESS: \\ Exploring the Productivity of Weak Architecture
}

Loretta Kong, B.A.S.

A thesis submitted to

The Faculty of Graduate Studies and Research

In the partial fulfillment of the requirements

For the degree of

Master of Architecture

Carleton University School of Architecture

Ottawa, Ontario, Canada

(C) 2005, Loretta Kong 


\section{Abstract}

This thesis proceeds in a groundless theoretical context, where architects are no longer able to construct on the basis of a universal epistemology, but must instead posit at every step their own goal and grounding. ${ }^{1}$ As a result, contemporary architectural experiences are produced at discreet and heterogeneous points to be assembled by each architect as a singular exploration of the material world, linked only by virtue of a connection to the human body.

This thesis explores the visceral encounter as a measure of contemporary architecture through the writing of architectural theorist Ignasi de Sola-Morales and twentieth century philosopher Henri Bergson. In particular, Sola-Morales's essay "Weak Architecture" contends that the strength of architecture emerges out of potent visceral exchange and not a strong conceptual base. This notion that knowledge of the material world emerges out of individual bodily engagement, is further explored in conjunction with Bergsonian thought, where memory and selfconscious reflection play a critical role in shaping the whole of human knowledge.

As a procedural grounding with which to investigate Sola-Morales's notion of weak architecture, this project is developed on the basis that built form cannot induce definitive experience, but that potent experience can only emerge out of self-reflective encounter. The investigation itself emerges out of a collaborative event to produced in conjunction with Ottawa's Le Groupe Dance Lab. This is subsequently explored through 1:1 construction and ultimately developed into a collaborative laboratory for experimental theatre. Instead of beginning with a fixed concept for architectural exploration, the work itself expands upon a temporal act as a conceptual grounding for the design project that follows, a grounding that is rationally weak.

\footnotetext{
' Ignasi de Sola-Morales, "Weak Architecture," trans. Graham Thomson, Differences: Topographies of Contemporary Architecture (Cambridge: The MIT Press, 1999) 58.
} 


\section{Table of Contents}

\subsection{Introduction}

\subsection{Deciphering Weak Architecture}

2.1 Weak theory

2.2 The End of Universal Subjectivity

2.3 Four Currents of Subjective Experience

\subsection{Henri Bergson and the Temporal Exchange}

3.1 Reason as Representation

3.2 Memory and Becoming

3.3 The Logic of Invention

\subsection{Personal monuments}

4.1 Precedent: Circus or the Caribbean Orange

4.2 Precedent: Holocaust Memorial

\subsection{An Investigation in Five Acts}

5.1 Act One: Dance Steps

5.2 Act Two: The Interdisciplinary Collaboration

5.3 Act Three: The Dance Machines

5.4 Act Four: Drawing From Memory

5.5 Act Five: Building the Laboratory

\subsection{Conclusion}

\subsection{List of Illustrations}

\subsection{Bibliography}




\subsection{Introduction}

This thesis contends that the contemporary aesthetic of architecture cannot be deciphered by any conceptual means, and is therefore rooted within visceral selfconsciousness. This notion will be explored through Ignasi de Sola-Morales's essay "Weak Architecture" and the writing of twentieth century French philosopher Henri Bergson.

In his essay entitled "Weak Architecture," architect and theorist Ignasi de Sola-Morales attempts to unravel the complexity of the modern experience by way of weak thought. The essay is proposed as a "diagonal cut, slanting, not exactly as a generational section but as an attempt to detect in apparently quite diverse situations a constant that seems to... uniquely illuminate the present juncture." ${ }^{2}$ This constant is a condition of groundlessness in the contemporary artistic landscape; a constant with paradoxically infinite permutations and infinite interpretive potential. Sola-Morales's response to this multiformity is the notion of weak architecture, a call to disassociate architecture from a foundation of totality and to accept the relegation of aesthetic practices to a secondary position of weakness.

It is impossible to ignore that the essay is as elusive as the condition of groundlessness it describes. The discussion self-consciously aims at edification

\footnotetext{
${ }^{2}$ Ignasi de Sola-Morales, "Weak Architecture," trans. Graham Thomson, Differences: Topographies of Contemporary Architecture (Cambridge: The MIT Press, 1999) 58.
} 
without instruction. In this way, Sola-Morales explicitly denies the possibility that his writing will develop into a formal strategy for weak architecture. Thus, at the end of the essay the reader is left with a sense of the condition, but no clear direction to build upon. In this way, the essay does exactly what it sets out to do. Instead of offering totalizing suggestions for our condition of groundlessness, Ignasi de Sola-Morales leaves us with an ambiguous definition of weakness that can be interpreted in a multitude of ways. Yet, for this very reason, the discussion on weakness raises questions regarding the dialogue between theory and architecture at large. By leaving the notion of weak architecture open to endless speculation, Sola-Morales challenges the productivity of theory itself. If theory, by conventional definitions, is a set of speculative beliefs that lead to a course of action, the notion of weak architecture itself challenges at every turn the possibility of building weak architecture. In effect, Sola-Morales's theory is itself a weak link to architecture.

By denying the reader an explicit link between the theory and built expression, Sola-Morales effectively shifts the strength of the essay away from his own voice as author into the hands of the reader as an interpreter. This is the premise of weak architecture. Because architecture cannot be contained within the confines of rational theory, the potency of architecture remains at its surface, in the space of encounter between subject and built form. Ultimately, the weakness of theory shifts the project of creating relevant architecture away from 
the rational constructs of intellectual thought and into the realm of corporal understanding.

The first section of this paper will focus on interpreting Ignasi de SolaMorales's discussion on weak architecture beginning with Gianni Vattimo's notion of weak thought as a precursor to "Weak Architecture." This is followed by an evaluation of "Weak Architecture" in four factors of subjectivity identified by Sola-Morales himself: the archaeological, the temporal, the decorative, and the monumental. The next section will explore the visceral exchange through Bergsonian thought, and is divided up into three main points. Firstly, that the intellect in its purity can only decipher to world by representing in the past tense and is therefore unable to understand the temporal exchanges that inform life. Secondly, that personal memory is critical in shaping the way we assemble meaning in all that we know, and thirdly, that the self-conscious faculty of intuition is our only means of expanding the boundaries of human knowledge itself. The third section of the discussion focuses on two precedents, Gordon Matta-Clark's Circus or the Caribbean Orange (1978) and Peter Eisenman's recent Holocaust Memorial in Berlin (2005). Both of these will be discussed in terms of Bergson and Sola-Morales thoughts on memory and monumentality.

Following this theoretical discussion, the project is established on the basis that built form cannot induce definitive experience, but that potent experience can only emerge out of self-reflective encounter. Instead of attempting to effect an experience upon a subject, this project aims at delaying the 
moment of encounter by interrupting the habitual to therefore arouse selfconsciousness in the individual. The investigation itself emerges out of a collaborative event to produced in conjunction with Ottawa's Le Groupe Dance Lab. This is subsequently explored through 1:1 construction and ultimately developed into a collaborative laboratory for experimental theatre. Instead of beginning with a fixed concept for architectural exploration, the work itself expands upon a temporal act as a conceptual grounding for the design project that follows, in an attempt to produce architecture that embraces strength in its weakness. 


\subsection{Deciphering Weak Architecture}

\subsection{Weak theory}

Ignasi de Sola-Morales' notion of weak architecture is based on philosopher Gianni Vattimo's theory of "weak thought." The basic premise of this theory is the idea that there is no longer a single, normative and universal foundation for philosophical debate and this has resulted in a crisis of reason. Vattimo introduces "weak thought" as an alternative to "strong thought" that is characterized by a search for a rational, comprehensive epistemology.

In his book The End of Modernity, Vattimo attributes the failure of the foundational system of reason to the absence of true objectivity. For instance, as opposed to the common conception that science is built upon objective truth while art is allowed to evolve freely, Vattimo argues that science is built upon the same paradigms as art, all of which are based upon a concordance of need, a perceived "functionality" in regards to life ${ }^{3}$. Like art, scientific paradigms emerge out of rhetoric, out of persuasion rather than demonstration. The author writes, "It occurs through a complex system of persuasion, active participation, interpretations and answers which are never exclusively nor principally the effects of force and violence, but involve a kind of aesthetic, hermeneutic, or rhetorical

\footnotetext{
${ }^{3}$ Gianni Vattimo, The End of Modernity: Nihilism and Hermeneutics in Postmodern Culture, trans. Jon R. Snyder (Baltimore: The John Hopkins University Press, 1991) 92
} 
assimilation." Even though science develops in a cumulative and seemingly processional way, it rests upon a foundation of values that are always changing. Science is only stable within its own mechanism. Regardless of the amount of scientific data humans have accumulated throughout history, science only progresses at a rate relative to the values of those scientists that perform the research. Hence the whole of science is built upon a body of experience that is always shifting.

Ignasi de Sola-Morales translates Vattimo's notion of weak thought into a weak theory for architecture. Instead of assigning a procedural grounding with which to create weak architecture, the essay self-consciously aims at edification without explication. "Weak Architecture" is itself a weak link to the actual, a discussion about the landscape of contemporary architecture that resigns itself to a position of weakness, thereby placing strength into the hands of the reader. More specifically, instead of proposing a formal strategy for weak architecture, SolaMorales' proposal is a "diagonal cut, slanting, not exactly as a generational section but as an attempt to detect in apparently quite diverse situations a constant that seems to...uniquely illuminate the present juncture." ${ }^{, 5}$ Its purpose is not to suggest formal intervention, but to trace a weak line of thought through the diverse terrain of architectural experience, that may lead a reader to interpret the built world in a way that is personal. Ultimately, weak architecture is weak by

\footnotetext{
${ }^{4}$ Vattimo, The End of Modernity, 92.

${ }^{5}$ Sola-Morales, "Weak Architecture," 58.
} 
virtue of weak theory, and contends that the potency of architecture is in the strength of visceral impact, not in its words and representations.

\subsection{The end of universal subjectivity}

Instead of assuming that an architectural experience can be contained within theory and rational concepts, weak architecture embraces the strength of architectural constructs in the complexity of the human experience. This discussion begins with the disappearance of an absolute frame of reference--the product of the classical imagination--and the convoluted journey to rediscover an objective reference point rooted in universal order. The failure of that project has produced what Ignasi de Sola-Morales calls the real crisis of the modern project that is the "situation of our contemporaneity." Faced with the impossibility of building from a universal system, architects and artists are confronted with a situation of groundlessness, in that every project holds "the obligation to posit for every step both its goal and its grounding.",

In a way, we can say that the loss of an absolute frame of reference or the 'death of God' is actually the death of the universal subject. In God, every citizen had an understanding of their own position in relation to an absolute. Every aspect of the temporal realm was also inherently related to this same reference point, thus "the world of architecture...effectively represents a vision of a closed

\footnotetext{
${ }^{6}$ Sola-Morales, "Weak Architecture," 59.

${ }^{7}$ Sola-Morales, "Weak Architecture," 59.
} 
and complete universe as a finished totality." ${ }^{\circ}$ Under this assumption, every creative act is one that elaborates upon an already existing unity, and through mimesis, each architect continues to create by representing this harmony. While the dissolution of God destroyed the paradigm of universal harmony, architects have continued to search for this missing source of order. In effect, they are searching for the universal subject by way of thought and theory.

Architectural theories, for the most part, are created on the basis of a kind of fictional subjectivity. For instance, Le Corbusier's personal manifesto Towards a New Architecture extolled the beauty of form that was defined by an absolute function in mechanical perfection. The fictional subject assumed is one that found beauty in the streamlined aesthetics of the automobile, the airplane, and the steamship. Le Corbusier's theory was, in effect, a hypothesis set forth by an architect to assume specific relationships between a subject and a physical environment. It is a way of prescribing values upon a subject in order to design upon an ideological grounding. Ignasi de Sola-Morales discusses the problems of such ideological methods in "Weak Architecture," specifically the fundamentalism of the modernist tradition and Kenneth Frampton's theory of critical regionalism. For the former, Sola-Morales discusses the New York Five's call in the 1960 's to reestablish a line of orthodoxy and correctness in the form of early twentieth century modernity and Richard Meier who continues to repeat the linguistic tropes of twenties purism. Both of these examples, according to Sola-

\footnotetext{
${ }^{8}$ Sola-Morales, "Weak Architecture," 58.
} 
Morales, amount to nothing more than pure historicism. Kenneth Frampton's critical regionalism, a claim for a new vernacularism, is also called into question. Although he praises Frampton's recognition that a 'system' is no longer possible, he questions Frampton's faith in a past order in which building, dwelling, and thinking were considered a unity. Even though Frampton does not provide explicit formal instructions for critical regionalism, Sola-Morales argues that his categories are merely attempts at restoring an old order that has little or no sense of the contemporary crisis. In other words, Frampton's theory assumes a fictional inhabitation in much the same way as those he criticizes.

Because the above examples are created on the basis of some kind of ideological fiction, they admit the possibility of control. What "Weak Architecture" essentially calls for, is the recognition that architectural experience should not be created under any projected ideas of inhabitation; that architecture should not be directed, it should be created only within experience itself, within the immediate, the now. Hence, weak theory is the key to weak architecture. In contrast to the nostalgic theories, weak architecture is a theory about thinking and making that neither identifies a way to think nor a way to make. Rather, it identifies prominent markers in the contemporary aesthetic without explicit indication as to what they are or where they may lead you. Weak architecture is essentially an essay on the architectural aesthetic. The aesthetic, as the term was used in the eighteenth century, was a philosophical study of the beautiful that 
originated from the Greek term aisthetikos, meaning "of sense perception." ${ }^{9}$ So the philosophy of aesthetic was directly linked to a study of the concrete and the encounter that occurs between the surfaces of the world and the subject that experiences it. As Sola-Morales points out, the aesthetic was "far removed from any pretensions to the totality of the ontological system." ${ }^{\prime 10}$ By weakening the link between the realm of ideation and the realm of experience, the potency of the aesthetic in the practice of architecture becomes more relevant now than ever before. Ignasi de Sola-Morales's main question in "Weak Architecture" is exactly this: "What role is accorded to architecture in the aesthetic system of weak thought?"

"Weak Architecture" is written precisely in the space between a subject and an object. Rather than commenting on the nature of an experience, or the other option, the nature of the form that shapes experience, Sola-Morales attempts to identify the properties that define the exchange. Additionally, Sola-Morales recognizes that his own writing is merely one interpretation of many possible interpretations of weak thought, and declares that it is not a "generational section," but an attempt to "uniquely illuminate the present juncture."11 The rest of the essay is written with the same amount of precision, as Sola-Morales delineates the boundaries of weak architecture with care not to ground the theory as an absolute. In this respect, the boundaries of weak architecture are traced

\footnotetext{
${ }^{9}$ James Smith Pierce, From Abacus to Zeus (New Jersey: Prentice Hall, 1998) 2.

${ }^{10}$ Sola-Morales, "Weak Architecture," 60.
} 
within the sands of subjective experience; they are temporary markers that draw attention to currents, not certainties. They are neither firm rules for construction, nor are they simply indecisive musings. This next section will explore four currents of thought identified in the writing, the archaeological, the temporal, the decorative, and monumentality.

\subsection{Four currents of Subjective Experience}

The archaeological is an analogy used to describe the superimposed readings of our reality in an aesthetic system, where reality is no longer read as a whole, but as a body of overlapping layers. ${ }^{12}$ By virtue of the elimination of temporal linearity and latent meaning, all objects exist on the same ideological plane, to be organized only by subjective experience in real time. Like the archaeological process of unearthing, collecting and interpreting forgotten objects, artists and architects interpret tectonic reality to an end that can be neither confirmed nor denied. Hence, readings of a work of art or architecture hinge upon the sensual subjective implications of the project. Meaning can only come from the outside of architecture, in the encounter between subject and object. As Sola-Morales states, "The signified is not constructed by means of an order but by means of pieces that may ultimately touch; that approach one another, at times without touching; that draw nearer to one another yet never make contact; that overlap,

\footnotetext{
"Sola-Morales, "Weak Architecture," 58.

${ }^{12}$ Sola-Morales, "Weak Architecture," 65.
} 
that offer themselves in a discontinuity in time whose reading as juxtaposition is the closest approximation of reality at our disposal."13 Like archaeological narratives, our systems are mental constructs that are created by our ability to string together discreet points, to create relationships as we see them, as opposed to discovering preexisting ones.

As Sola-Morales recognizes, the notion of the archaeological and the fragmentary seem to imply a simple translation into built form. One can imagine that the formal expressions of postmodernism aimed for experience that is complex and fragmentary, with similar intents to dissect a modern experience that was no longer total. What began as a critical rejection of the new in postmodernism turned into a formal representation of the tenets themselves, rooted not in experience, but in formal games with historical pastiche. Under the assumption that the fragments themselves were meaningful, much of post modernist work developed into a cut and paste of historical form. Ultimately this denied the possibility for relevant contemporary architecture without reaching into cultural presets of once significant forms. This is precisely the mistake that that Sola-Morales attempts to avoid. To illustrate this point, he writes, "Nobody can be so naïve as to imagine that, for archaeology, the system of knowledge of the past can be constituted by a simple accumulation of the objects uncovered by excavation." ${ }^{\prime 14}$ Because objects of art or architecture are unable to contain their

\footnotetext{
${ }^{13}$ Sola-Morales, "Weak Architecture," 66.

${ }^{14}$ Sola-Morales, "Weak Architecture," 66.
} 
own meanings, the fragmentary in Sola-Morales's case lies not behind the surfaces of the building but in front of them, the layering of meaning in the archaeological occurs in the body, not in the form.

The hierarchical flattening of the world demands an understanding of time that is neither a structured system nor a progression to an end, but a point of coincidence. A reading generated through superimposition can only come into fruition when the outcome cannot be anticipated. Experiential time is personal and subjective. Because the fragmentary meanings of the archaeological are assembled only within the now, the momentary 'coagulation' of experience can only occur within the present. In this way, an aesthetic experience can never be translated into a language of forms; it is necessarily rooted in the temporal event. Sola-Morales writes, "[t]emporality does not present itself as a system but as an aleatory instant that, responding above all to chance, is produced in an unforeseeable place and moment. In certain works of contemporary art, in dance, in music, in installation, the experience of the temporal as event, occurring once and then gone forever, ably explicates a notion of temporality that finds in the event its fullest form of expression." time, weak architecture is contingent upon subjective time, time that is felt, anticipated, or ignored. The experience of time is never simply counted.

Together, the fragmentary and the temporal form an experience that is subjective and objective at the same time. Instead of assuming that matter is 
ordered in some mysterious unknown way, the aesthetic of weak architecture places everything on the surface. Hence the physical environment can only be ordered by the individual that experiences it. As Sola-Morales writes: "Reality emerges as a continuum in which the time of the subject and the time of external objects go round together on the same looped tape, with the encounter of objective and subjective only occurring when this continuous reality folds over in a disruption of its own continuity."

The continuity of subject and object translates into a flattening out of the hierarchy of the world. For this reason, Sola-Morales places weak architecture within the inessential, the decorative. The decorative is traditionally identified as an unnecessary surface application affixed to the 'essential' structure of architecture that is often associated with taste. Sola-Morales is not advocating that architects be concerned with taste or that they become decorators. Rather he argues that if architecture is to be a study of the concrete, then all of matter is inessential, by virtue of the nonexistence of the essential. Hence, instead of dividing architecture into different measures of importance, an architect concerned with aesthetics should hold all aspects of the concrete in the same regard. This is neither a question of potency nor validity. Rather the elimination of strong universal values in architecture leaves the whole of architecture in a circumstance that was formerly understood as peripheral, where nothing is

\footnotetext{
${ }^{15}$ Sola-Morales, "Weak Architecture," 68.

${ }^{16}$ Sola-Morales, "Weak Architecture," 69.
} 
essential in regards to the universal, yet all is essential in regards to the aesthetic experience. Architecture is the inessential element of the concrete that forms our experiences, that enhances and embellishes reality, without at any moment turning into a fundamental truth or meaningless form.

This leads to the last characteristic that Ignasi de Sola Morales describes in respect to weak architecture, the notion of monumentality. As the embodiment of cultural memory, the monument is not an easy subject to discuss in regards to weak thought. Traditionally, monuments are built as grand signifiers to historical events, built universals. Ignasi de Sola-Morales states:

"The monument in the classical age is the center, it is the imago $D e i$, the figuration of a transcendent divinity that guarantees the consistency of time. The figure of the king in the middle of the Royal Square thus constitutes the emblem of the power that hierarchally orders a given public space. The obelisk at the central point of the perspective is the monument that guarantees the coherence and immovability of the representational visual structure. It is not about this monument that I wish to speak. The monumentality of weak architecture is not continuous with the monuments of the classical age in either geometric or ideological 
value, but only in what remains within the present context of the root term monitu; that is to say, of recollection."17

Sola-Morales challenges our traditional idea of monument as a representation of the absolute. Again, because of the impossibility of embedding universal meaning in built form, a weak monument cannot be a signifier of permanence, such as Aldo Rossi argues in his Architecture of the City. The monumentality of weakness refers back to the subject. It lies not within a form that can be seen, understood and forgotten, but within an experience that embeds itself in personalized memory. Personal recollection is the only form of memory that a monument can ever possibly create. He states: "the notion of monument that I have sought to put forward here is bound up with the lingering resonance of poetry after it has been heard, with the recollection of architecture after it has been seen." 18

In these four factors, Ignasi de Sola-Morales presents us with the understanding that weak architecture emerges from individually assembled experiences. The archaeological, the temporal, the decorative, and the monumental are all built upon the recognition that weak architecture does not exist prior to subjective experience, that meaning is not read off of the surface of built form. Rather, architectural meanings are formed on the outside by individual bodies in the moment of encounter.

\footnotetext{
${ }^{17}$ Sola-Morales, "Weak Architecture," 70.

${ }^{18}$ Sola-Morales, "Weak Architecture," 71.
} 


\subsection{Henri Bergson and the Temporal Exchange}

Before Gianni Vattimo's notion of weak thought, the weakness of human reason was discussed in the writings of twentieth century philosopher Henri Bergson. For Bergson, the crisis of reason is inevitable by virtue of our imperfect intellect. The search for a rational absolute is bound to fail because the foundation of life is ultimately irrational. Our only means of knowing about life is through the medium of temporal exchange between our bodies and the built world. The temporal exchange is the basis of three main points to be discussed. Firstly that human reason is inherently unable to understand life at the moment that it occurs. Secondly, that this inability to function within the present means that memory and recollection play a crucial role in shaping how we know our world, and lastly, because we are unable to definitively understand life by rational means, reason and action form a cycle of invention that is ever expansive, expanding the boundaries of what we know, consequently affecting the way we act in the world.

\subsection{Reason as Representation}

For Henri Bergson, the human intellect is our faculty for mechanical reasoning that dominates conscious thought. Although in actuality our intellect does not exist in absolute purity, for the purposes of this discussion we will begin with pure intellect. Bergson argues that pure intellect is inherently unable to understand life. Life always occurs within duration, defined as the unceasing 
heterogeneous flow of the present. Instead of operating within duration, pure intellect reduces experience into conceptual representations such as language and image. Essentially, it forms an artificial version of experience that is merely a weakened representation of the actual. Just as we are unable to represent accurately the experience of a city through the combined effort of photographs, videos and written description, the representation of our bodily experiences will never be the same as bodily sensations themselves. The intellectual process as a whole is an artificial approximation of the universe that fragments the whole into pieces of the past. Because life is a tendency towards the future, our pure intellect is ultimately characterized with the natural inability to comprehend life. Hence human reason, in and of itself, is incapable of reconciling our existence with the indeterminate nature of duration.

Bergson believes that pure intellect is merely a mechanism that allows us to proceed cautiously into the future and functions through analysis. "[T]o analyze... is to express a thing as a function of something other than itself. All analysis is thus a translation, a development into symbols, a representation taken from successive points of view from which we note as many resemblances as possible between the new object which we are studying and others which we believe we know already."19 In other words, analytical thinking, such as science, will always work with repetition as a method, it will always attempt to decipher

\footnotetext{
${ }^{19}$ Henri Bergson, An Introduction to Metaphysics, trans. T.E.Hulme, (Indianapolis and New York: The Library of Liberal Arts, 1955), 24.
} 
information in terms of what is already known. As opposed to operating within the moment in which 'life' happens, it operates only within "the discontinuous, in the immobile, in the dead." ${ }^{20}$ And although it is our tendency to prognosticate the unforeseeable though analysis, Bergson contends that this will never lead us any closer to approaching an absolute knowledge of life. So in this regard, the analytical search for the absolute is redundant. It is an attempt to find the absolute by rationalizing it to no end.

The supposition that the collective human intellect can acquire enough knowledge of the universe to thereby attain the absolute, is a fiction, "[o]ur reason, incorrigibly presumptuous, imagines itself possessed, by right of birth or by right of conquest, innate or acquired, of all the essential elements of the knowledge of truth. Even where it confesses that it does not know the object presented to it." ${ }^{21}$ Bergson's position is that although our pure intellect does not have the capacity to understand the fullness of human experience, it will continue to act as though it can. As a result, our pure intellect will tend to create a stable epistemology because it is not able to rationalize the flux of life. In this way the intellect seeks to harmonize a world that cannot be harmonized. This is Bergson's difficulty with finalistic views of the universe, in which human civilization is perceived to be progressing linearly towards a state of perfect harmony, when in fact, harmony itself is a human construct. Harmony is constructed by

\footnotetext{
${ }^{20}$ Henri Bergson, Creative Evolution, trans. Arthur Mitchell (New York: The Modern Library, 1944) 182.
} 
retrospective reflection upon the past, and by grouping singular experiences into seemingly natural groupings. Bergson states: "Harmony, or rather "complementarity," is revealed only in the mass, in tendencies rather than in states ... harmony is rather behind us than before. It is due to an identity of impulsion and not to a common aspiration." 22

According to Henri Bergson, life does not proceed linearly towards a point in the distant future, it expands along many lines that increase exponentially as time moves forward it evolves with life. He states: "The evolution movement would be a simple one, and we should have been able to determine its direction, if life had described a single course, like that of a solid ball shot from a cannon. But it proceeds rather like a shell, which suddenly bursts into fragments, which fragments, being themselves shells, burst in their turn into fragments destined to burst again, and so on for a time incommensurably long."23 Human experience builds only upon itself. Individual experience infinitely builds upon individual experience, expanding the collective consciousness into a cloud of fragments, impossible to connect. Because these fragments come from extraneous points, the direction of the future is impossible to foresee. This is quite different from a straight arrow path that ends in a state of rest. For Bergson, the human condition becomes more and more complex with time. Multiplicitous in nature, consciousness can only be reconciled in the equally complex context of the

${ }^{21}$ Bergson, Creative Evolution, 55.

${ }^{22}$ Bergson, Creative Evolution, 58. 
human body. An empirical understanding of the world always returns to the human body, and the present moment.

Because the Bergsonian absolute does not exist as a static reference point but as heterogeneity intricately bound within the passage of time, "the absolute, which is the object and not its representation, the original and not its translation, is perfect, by being perfectly what it is. ${ }^{, 24}$ The only means of access that humans have to acquire knowledge about life is through the medium of the body itself. As builders of new human experiences, architects have often struggled with the inability to create a rational ground for architecture. By recognizing that architectural meaning is generated by our experience of built form, and not the product of ideas translated into buildings, we unhinge objectivity from its once unshakable foundation. Hence, the project to create relevant contemporary experience becomes both the goal and grounding for the creation of architecture. The visceral itself is the measure of contemporary architecture.

\subsection{Memory and Becoming}

As opposed to a subject with a defined metaphysical foundation, the subject described in "Weak Architecture" is one that is in a perpetual state of incompleteness, or what Bergson refers to as "becoming."25 This is based upon

\footnotetext{
${ }^{23}$ Bergson, Creative Evolution, 109.

${ }^{24}$ Bergson, An Introduction to Metaphysics, 23.

${ }^{25}$ Henri Bergson, Matter and Memory, trans. N.M. Paul and W.S.Palmer (New York: Zone Books, 1988) 77.
} 
the understanding that an individual existence does not precede the physical world but emerges in continual exchange with it. Contrary to our understanding that space is a container for event, Bergson contends that space is a by-product of our continual exchange with the exterior. The nature of this expansive exchange has been explored in his seminal work Matter and Memory.

The basic premise behind Henri Bergson's discussion in Matter and Memory is that individuals do not have the power to shape their world, rather both the world and individual consciousness emerge out of continual exchange. The foundation of Bergson's theory, as we have mentioned before, is the notion of duration, the absolute, the ceaseless and heterogeneous flow of the present into the future. Our consciousness is a derivative of duration. Thus, like duration there are no ideas, feelings or volitions in our conscious state that are not undergoing change at every moment, although we are not always aware of it. As an example, Bergson describes the conscious perception of a motionless object as follows: "The object may remain the same, I may look at it from the same side, at the same angle, in the same light; nevertheless the vision I now have of it differs from that which I have just had, even if only because the one is an instant older than the other. My memory is there, which conveys something of the past into the present. My mental state, as it advances on the road of time, is continually swelling with the duration that accumulates: it goes on increasing - rolling upon 
itself, as a snowball on the snow." ${ }^{26}$ Just as time trudges on into the future, our conscious minds accumulate more and more experiences. According to Bergson, this change goes unnoticed until it is sufficient enough to impress a new attitude upon the body and redirect our attention to the fact that our state has been altered.

Instead of recording reality in its full and unadulterated state, Bergson believes that our perceptions are selectively influenced by memory. Memory shapes perception by interrupting it. The first effect of memory, is that of contraction. As opposed to the usual assumption that perception is a direct translation of everything we perceive, Bergson believes that our perception records a set of static states that are selectively contracted by our memory. $\mathrm{He}$ writes, "instead of a flux of fleeting shades merging into each other, it perceives distinct and, so to speak, solid colors, set side by side like the beads of a necklace; it must perforce then suppose a thread, also itself solid to hold the beads together. ${ }^{, 27}$ The thread that connects these beads is our own personal rendering of the moment, an artificial "formless ego" that is always a step removed from reality. In addition to this contraction, the "individual beads" themselves are also products of our memory. Each moment of external perception triggers discreet recollections from our past, described by Bergson as a "cloak of recollection." 28 The immediate data from our senses arouses discreet recollections from our past, creating composites of external data intermingled with thousands of details from

\footnotetext{
${ }^{26}$ Bergson, Creative Evolution, 4.

${ }^{27}$ Bergson, Creative Evolution, 6.
} 
our past experience. In many cases, our memories may even supplant the external data. A simple illustration is how two people can recall completely separate details of the same film and leave with very different impressions. Because we are constantly accumulating more experiences, no two perceptions of the same environment will ever be the same. "Memory, inseparable in practice from perception, imports the past into the present, contracts into a single intuition many moments of duration, and thus by a twofold operation compels us, de facto, to perceive matter in ourselves, whereas we, de jure, perceive matter within matter." 29

In this way, memory is not a faded perception, but the essence of consciousness. Memory supplements our pure external readings of the physical environment with virtual images of similar environments and situations. Hence, conscious perception is actually the coming together of present data collected by extending ourselves into objects, and a virtual survey of possible actions that our bodies can effect on the surrounding environment. In Bergson's model, the present is always an anticipation of impending future action that is formed in two parts, the actual physical domain of our bodies, and the virtual domain of memory that has no connection to our actual bodies. The past does not exist before the present, but in simultaneity with it. Along with this simultaneity, the different moments of the past are also distinguished from each other. Each successive bead

\footnotetext{
${ }^{28}$ Bergson, Creative Evolution, 5.

${ }^{29}$ Bergson, Matter and Memory, 74.
} 
of memory is distinguished from the one that precedes by both a measure of difference and repetition.

In Bergson's model, space is conceived in duration. As opposed to the more traditional notion that space precedes time and experience, Bergson's notion of space is one that unfolds and actualizes itself in motion:

Concrete extensity, that is to say, the diversity of sensible qualities is not within space; rather it is space that we thrust into extensity. Space is not a ground on which real motion is posited; rather it is real motion that deposits space beneath itself. But our imagination, which is preoccupied above all by the convenience of expression and the exigencies of material life, prefers to invert the natural order of the terms ... Therefore, it comes to see movement as only a variation of distance, space being thus supposed to precede motion. Then, in a space which is homogeneous and infinitely divisible, we draw, in imagination, a trajectory and fix positions: afterwards, applying the movement to the trajectory, we see it divisible like the line we have drawn, and equally denuded of quality. ${ }^{30}$

As architects, we are conditioned to regard space as an infinite divisible field that contains all the events that occur within it. But if we are to regard temporal experience as the foundation of space, then space is not a complete and uniform 
measure of our physical environment. Rather it is an artificial by product of a multitude of fleeting images, created in a continual state of becoming.

This heterogeneous state of becoming has been explored in disciplines outside of architecture. Ignasi de Sola-Morales's states: "[c]ontemporary time today's fragmented reality of overlapping virtual and "real" times that was artistically anticipated in the writings of James Joyce, Robert Musil, and Mario Vargas Llosa - is presented precisely as juxtaposition: a discontinuity; something that is in complete contrast to a single, unique, closed and complete system."31 As opposed to an abstract notion of time as a regular and unified progression into the future, contemporary time is imbued with memory and experience, it is interpreted, anticipated and ignored. Contemporary time has also been explored in literature, theatre, music and film. Time-based media such as these can explore perception of time by engaging a subject's expectation or memory of an earlier scene. Recent films such as Christopher Nolan's Memento $(2000)^{32}$, also explore time as a function of memory. In this case, the plot unfolds from the viewpoint of a protagonist who suffers from short term memory loss. By using tattoos and notes he attempts to hunt down his wife's killer. The plot is presented in chunks as long as the protagonist himself can remember and moves back in time, hence, each new scene generates a different interpretation of the previous. Although the exploration of changing perspectives is nothing new, a movie such as Memento

\footnotetext{
${ }^{30}$ Bergson, Matter and Memory, 217.

${ }^{31}$ Sola-Morales, "Weak Architecture," 67.
} 
explores the evolution of an event through the viewer's own recollection as well as that of the protagonist. A film such as "The Usual Suspects," ${ }^{33}$ in another example that engages the expectations and memories of the viewer. Much of this movie is told from the point of view of the protagonist Roger 'Verbal' Kint at an inquisition conducted by U.S. Customs Special Agent David Kujan. He is one of two witnesses that survived the explosion of a boat believed to have over $\$ 91$ million dollars worth of cocaine on board. The movie unfolds as Kint recounts the events leading up to this explosion. This movie ends with a revelation that forces the viewer to recall all that was previously viewed and understood. This narrative virtually folds back upon itself, causing the viewer to reconsider the entire movie. By exploring the element of memory and recollection in our understanding of contemporary time, reality is rendered flexible, a malleable surface that is shaped by memory, recollection, and cognition.

\subsection{The logic of invention}

Up until this point, we have discussed pure intellect in order to understand the tendencies of the human intellect. But in actuality, intellect and instinct form divergent forces of the same consciousness, acting to propel human nature through an ever-expanding logic of invention. In isolation, the human intellect is unable to transcend itself because it will be forever shut up in a cycle of the given.

\footnotetext{
${ }^{32}$ Memento, dir. Christopher Nolan. Newmarket Film Group, 2000.

${ }^{33}$ The Usual Suspects, dir. Bryan Singer. Metro-Goldwyn-Mayer, 1995.
} 
As we have discussed previously, human reason by itself is only capable of understanding the past, and not the present ${ }^{34}$. Similarly, Bergson asserts that instinct by itself is trapped within the cycle of life, in a continual state of the present, incapable of self-reflection. Ultimately, it is the self-conscious acknowledgement of both in intuition that allows us to reflect upon the infinitely expansive duration of life.

Bergson differentiates instinct by intrinsically linking it through motion to the body. In contrast to the intellect, which is incapable of operating within the indeterminacy of life, instinct operates within the precise moment of the present in which life happens. In its purity, the instinct is a visceral understanding of the world, action incapable of representation. Organisms that are ruled by instinct function in a highly complex yet completely organized manner, their responses to matter are innate yet seem very rational. For example, insects do not stop to ponder the direction of their lives, they simply live. They act and react to their environment in the present because it is the only way they know how. In this way, instinct is exterior to all images and representations. A purely instinctual reaction bypasses self-awareness, it has no need or capacity to question its own purpose. As a result, instinctual societies are unified, stable and closed systems. Bergson uses as an example the societies of ants and bees, which are entirely organized around a common purpose. Each insect has a role to serve and will serve that exact purpose, with no propensity to change its fate. Therefore, an

\footnotetext{
${ }^{34}$ Bergson, Creative Evolution, 180.
} 
instinctual society is unified and harmonious in nature. Organisms ruled by instinct are unable to seek or imagine alternatives by virtue of their inability to leave the exact moment of the present.

Similar to insects, human instinct is also linked to motion, with the difference being that human self-consciousness is never completely absent but is temporarily nullified by action. For instance, "[w]hen we mechanically perform a habitual action, when the somnambulist automatically acts his dream, unconsciousness may be absolute; but this is merely due to the fact that the representation of the act is held in check by the performance of the act itself, which resembles the idea so perfectly, and fits it so exactly, that consciousness is unable to find room between them. Representation is stopped up by action." ${ }^{35}$ Hence, to know something instinctually, is to now how to react to it, to know how to use it without question, without reflecting upon its nature and purpose. For humans, action lapses back into the rational realm when alternatives emerge. When a decision needs to be made, humans essentially step outside actual action into the virtual realm of potential action to weigh the pros and cons of known possibilities.

Instead of depending upon innately unified reactions, the intellect allows us to see possibility within matter. Unlike the insect that responds to stimulus in the only way it knows how, a person sees possibility outside of the moment. For instance, "A form, just because it is empty, may be filled at will with any number 
of things in turn, even with those that are no use. Formal knowledge is not limited to what is practically useful, although it is in view of practical utility that it has made its appearance in the world." ${ }^{36}$ Yet, precisely because the intellect operates by virtually reconstituting matter, the possibilities that are considered will always be assemblages of what we already rationally understand. The rational evaluation of a present circumstance will still lead us no closer to understanding the unforeseeable relationships between our bodies and the world. For this, we must rely on our intuition.

According to Bergson, intuition is what allows us to transcend both cycles of instinct and intellect by way of sympathetic communication with the body. $\mathrm{He}$ defines intuition as instinct that has become self-conscious, able to reflect on its object yet continue to act within it, He states:

On the one hand, it will utilize the mechanism of intelligence itself to show how the intellectual molds cease to be strictly applicable; and on the other had, by its own work, it will suggest to us the vague feeling, if nothing more, of what must take place within the intellectual molds. Thus, intuition may bring the intellect to recognize that life does not quite go into the category of the many nor into that of the one; that neither mechanical causality nor finality can give a sufficient interpretation of the vital process.

\footnotetext{
${ }^{35}$ Bergson, Creative Evolution, 159.

${ }^{36}$ Bergson, Creative Evolution, 167.
} 
Then, by the sympathetic communication which it establishes between us and the rest of the living, by the expansion of our consciousness which it brings about, it introduces us into life's own domain, which is reciprocal interpenetration, endlessly continued creation ... Without intelligence, it would have remained in the form of instinct, riveted to the special object of its practical interest, and turned outward by it into movements of locomotion. ${ }^{37}$

Bergson argues that intuition is able to transcend the cycle of intellect and intuition by virtue of its connection to life. While consciousness is simply the capacity to perceive, observe and respond to the world around us, intuition is informed by a self-conscious visceral awareness of one's own body. Thus, to intuit is to know the world by listening to one's body, to engage in "the art of intellectual auscultation." ${ }^{38}$

Self-conscious reflection by intuition is the basis by which we can extend the boundaries of our known world. Because "it is of the essence of reasoning to shut us up in the circle of the given," 39 action allows us to break this circle. To illustrate this concept, Bergson uses the example of swimming. Suggesting that if we have never seen a man swim, we may suppose the act of swimming to be impossible. Yet, if we simply hurl ourselves into the water we may stay afloat by

\footnotetext{
${ }^{37}$ Bergson, Creative Evolution, 195.

${ }^{38}$ Thomas A Goudge, "Editor's Introduction," in An Introduction to Metaphysics, trans.

T.E.Hulme, (Indianapolis and New York: The Library of Liberal Arts, 1955) 13.

${ }^{39}$ Bergson, Creative Evolution, 211.
} 
struggling, and in this way become adapted to our new environment. Reason will always attempt to nail us down to a solid grounding, whereas action can propel us into the unknown. Following the precedent set by the very first swimmer, the rational assessment of risk decreases, and continues to decrease as more people begin to swim. In this way, the initial spark of action allows us to move beyond the boundaries of accepted reason into new territory. By leaping into the new territory that is beyond the edge of our perceived reason, we can extend our the reach of reason.

In effect, the ineffable momentum of our intuition forms basis for all knowledge of the material world. Without this sensitivity towards our bodies, we would be unable to extend our rational base. By contending that self-conscious intuition is our most immediate interface with the absolute, Bergson, like SolaMorales, emphasizes that our individual understanding of the visceral is the root of all understanding in human culture. Our personal tendencies which are informed by our unique experiences become the grounding that all of human knowledge rests upon. Although, rationally, it appears to be in constant flux, this subjective grounding, is stabilized by virtue of its connection to our bodies that endure in the constant, ceaseless duration of temporality. 


\subsection{Personal Monuments}

Two precedents will be discussed in reference to the discussion up until this point. First is Gordon Matta-Clark's project Circus or the Caribbean Orange, a subtraction that, by all accounts, demonstrates a an evolving interface with time that is rooted in subjective experience. The second project is the recently constructed Holocaust Memorial by Peter Eisenman. This discussion will focus more upon how the turn to individual, subjective experiences have changed the way we approach the public memorial.

\subsection{Precedent: Circus or the Caribbean}

\section{Orange, Chicago, 1978}

In January of 1978, Gordon Matta-Clark made three spherical cuts in a building adjacent to the Museum of Contemporary Art in Chicago ${ }^{40}$. This site, now destroyed, was slated for demolition the following year as part of the museum's expansion. The project was the result of an invitation from then curator Judith Russi Kirshner to operate on a three storey brownstone, it was the last project Matta-

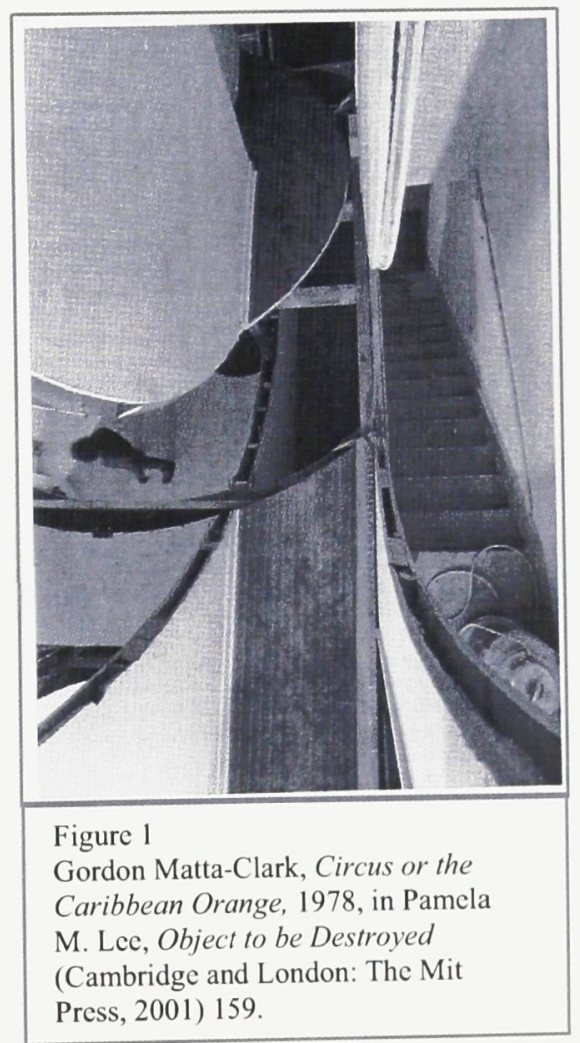

${ }^{40}$ Pamela M. Lee, Object to be Destroyed (Cambridge and London: The MIT Press, 2001) 142. 
Clark completed before his death in the same year. Despite its brief existence, the operation, titled Circus or Caribbean Orange, made an impression on artists and architects alike and continues to generate discussion today, almost thirty years later. In her book Object to be Destroyed, art historian Pamela M. Lee discusses this work in terms of a "heuristic sublime." ${ }^{41}$ Unlike the notion of sublime that begins and ends with an external spiritual grandeur, Lee suggests an experience of awe that leads to a kind of personal discovery. Lee's own illustration of the operation depicts a kind of temporal dynamism that transpires as one moves

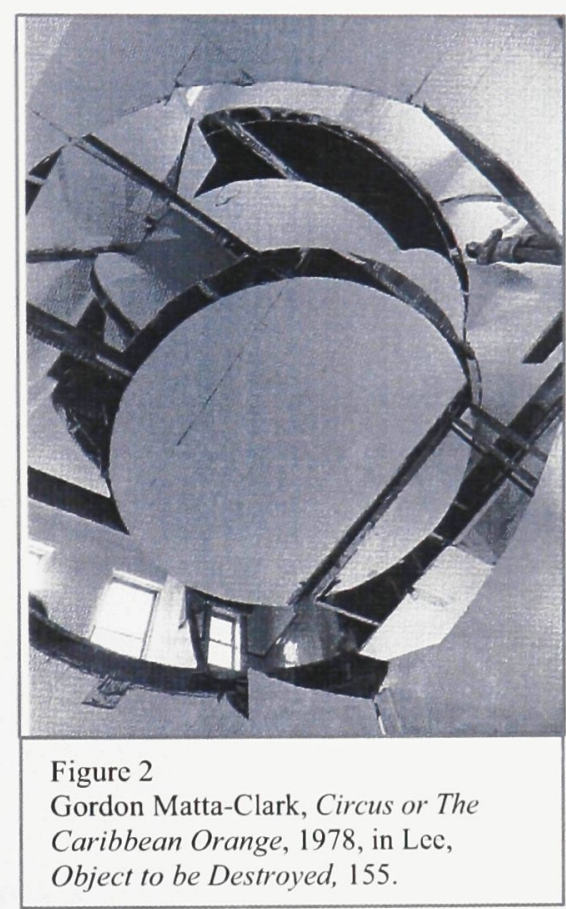
through the piece:

This kind of dynamism, experienced through the "confrontation with real time,' is not that of the subject strolling casually, easily, through phenomenological space. We are far away from the free plan here; if anything, we have now given way to a free fall. For the artist characterized the movement through his work as the most embodied of dynamics, so much so that the viewer "can't understand" the experience. The litany of reactions invoked by Matta-Clark - quake, gag, spasm - are bodily impulses that arise 
from the subject's mental negotiation with real time and space, corresponding to the subject's literal passage within the building. Alternately halting and kinetic, it suggests a virtual seizure of the subject consumed by a violent force. The almost schizophrenic character of this encounter can be related generally to Kant's formulation of the dynamical sublime. The time of the object, at once 'all consuming' and yet 'gagging,' begs the temporal integration or coherence of the viewer's experience. ${ }^{42}$

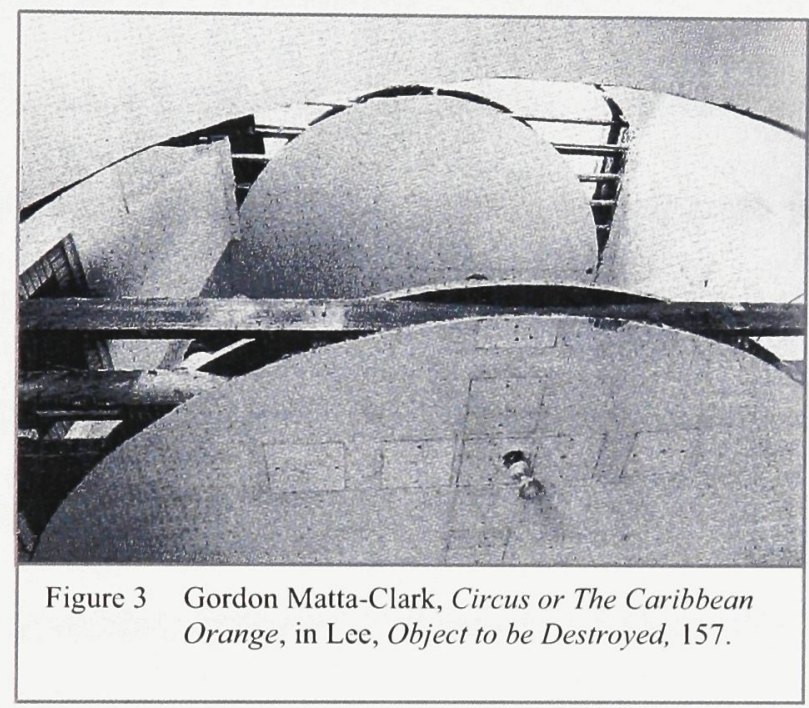

Lee describes an encounter in time characterized by the overwhelming desire to 'see more,' to 'experience more.' This desire entices a subject to move around the cut, to engage the cut by gathering different perspectives. The inability to understand without moving leads to a violent desire to decipher, a bodily impulse to possess the work, to seize it from the realm of the abstract.

\footnotetext{
${ }^{41}$ Lee, Object to be Destroyed, 138.

${ }^{42}$ Lee, Object to be Destroyed, 154.
} 
The potency of Matta-Clark's operations will never be fully understood by those of us that have never experienced it. Yet, the drive to move around and decipher the subtraction is possibly a result of the discontinuity of its form. Despite the formal minimalism of Matta-Clark's cuts, it is still difficult to grasp in experience because it defies habit. Unlike our buildings which are defined by a series of positive spaces that allow us to navigate freely, the spherical sections in Circus are not defined by continuous form, but by continuous absence. Navigation is the assembly of fragments in an attempt to connect the visible through what is invisible. For instance, because the 'form' of the sphere cannot be read unless it happens to intersect a floor or a wall, a viewer needs to draw invisible lines through space in order to connect disparate fragments. One cannot physically move within the moments of intersection between the virtual sphere and the actual structure, making the challenge to imagine connections thorugh memory images. Without a summarized view of the sphere in totality, the viewer is challenged to assemble fragments mentally. Essentially, the piece becomes an exercise in assembling recollections, of connecting where you've been with where you are by way of temporal superimposition. Because each person brings a different perspective, a different viewpoint, each experience is unique. MattaClark stated: "I don't know if you can read the negative space." 43 For the very reason that the cut cannot be read, it is reassembled, reflected upon and ultimately interpreted by those that have engaged it.

${ }^{43}$ Lee, Object to be Destroyed, 157. 
The result is a plethora of personal accounts that range from feelings of vertigo, ${ }^{44}$ to analogies between the act of cutting and the body in dance ${ }^{45}$. One viewer even noted that the piece explored figure-figure relationships as opposed to figure-ground. ${ }^{46}$ Circus or Caribbean Orange was created with no pretensions in regards to function, permanence, or monumentality. Because this was a temporary installation slated for inevitable demolition, Matta-Clark's requirements were different from those of a functioning building. Instead of focusing on creating static spaces read by static bodies, he created a space that could not be read unless one was moving. The artist's intention was simply to create a "circle in which you circle — a place of activity, a circle for action." ${ }^{47}$ By focusing on the experience of the space through movement, the experience opens up to possibility. Matta-Clark observed: "The thing I would really like to express is the idea of transforming the static, enclosed condition of architecture on a very mundane level into this kind of architecture which incorporates... this sort of animated geometry or this animated, tenuous relationship between void and surface ... [It] implies a kind of kinetic, internal dynamism of some sort." ${ }^{48}$ This kind of dynamism is only possible when subject and object merge.

In Gordon Matta-Clark's work meaning cannot be arrived at independently from personal recollection and interpretation. Instead of perceiving

\footnotetext{
${ }^{44}$ Lee, Object to be Destroyed, 157.

${ }^{45}$ Lee, Object to be Destroyed, 136.

${ }^{46}$ Lee, Object to be Destroyed, 158.

${ }^{47}$ Lee, Object to be Destroyed, 153.
} 
the cut in totality, the space of the cut can only be unfolded to the viewer in an intimate, private exchange. Circus does not, at any point divorce the relevance of the work from the temporal realm. To perceive Circus is to recall it in its various pieces, never to understand it as an abstract whole. Circus is a project that operates within the Bergsonian premise that the perceiving subject and the perceived object are in a constant state of becoming. Architecture does not, in and of itself, contain memorable events. Rather space is formed within the continual successive exchange between an individual and the external environment. This notion of space is vastly different from the "meaningful" objective spaces of modernism and postmodernism. Circus demonstrates by all accounts, that spatial significance is a matter of subjective, personal encounter. .

\subsection{Precedent: The Holocaust Memorial, Berlin, 2005}

This discussion would not be complete without returning to the notion of monumentality. The traditional monument is antithetical to all that we have discussed so far. Based upon symbolism and representation, the traditional notion of monumentality assumes that personal experience has nothing to offer the collective, that collective meaning must arise from a conceptual framework of symbol and representation. As we discussed briefly in a previous chapter, Ignasi de Sola-Morales asserts that the strength of the monument comes from personal recollection. The 'weak' monument is "bound up with the lingering resonance of

${ }^{48}$ Lee, Object to be Destroyed, 153. 
poetry after it has been heard, with the recollection of architecture after it has been seen." ${ }^{49}$ As opposed to assuming that collective meaning can only arise through the employment of already existing concepts, Henri Bergson and Ignasi de Sola-Morales both argue that the collective is formed by the accumulation of individual experiences, that the potency of collective memory begins with a potent individual experience. This section will discuss the notion of monumentality in regards to weakness by comparing two monuments, Peter Eisenman's Holocaust Memorial in Berlin, and the Canadian War Memorial in Ottawa.

Traditional monuments are essentially built attempts to freeze time. Monuments such as "the figure of the king in the middle of the Royal Square," ${ }^{50}$ and "the obelisk at the central point of the perspective" ${ }^{15}$ were constructed as a tectonic embodiments of public memory, built with the intent of preserving a pocket of time within the hearts and minds of all the public. For instance, Ottawa's National War Memorial was built to commemorate war by representing it. This

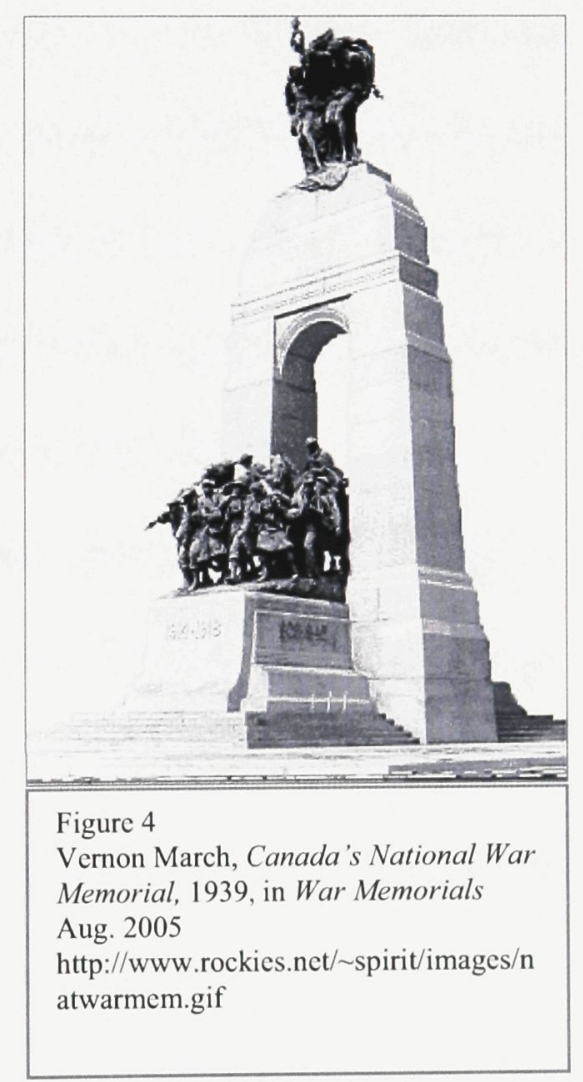

\footnotetext{
${ }^{49}$ Sola-Morales, "Weak Architecture," 71.

${ }^{50}$ Sola-Morales, "Weak Architecture," 70.

${ }^{51}$ Sola-Morales, "Weak Architecture," 70.
} 
monument by artist Vernon March was built to commemorate all of Canada's war dead by providing an icon that, theoretically, all citizens can relate to on an equal basis. Essentially, such a memorial externalizes the event to the point of view of the third person. The memorial depicts a troop of twenty-three anonymous bronze soldiers representing different arms of the service, marching through an archway. Inscribed on the sides of the archway are the dates "1914-1918" for the First World War, "1939-1945" for the Second World War, and "1950-1953" for the Korean War. While the soldiers are depicted at a one to one scale, their bodies are lifted above the street, causing onlookers to look up to the soldiers, both physically and metaphorically. The viewer is intended to hold the accomplishments of the soldiers in great esteem, thereby separating his or her own life from the lives of those that fought and died in the war. This kind of monument attempts to convey a message that is objectified, that refers only to the events that it memorializes, with limited room for personal interpretation.

Monuments such as the Canadian National War Memorial attempt to imbue form with inherent conceptual meaning upon conception. In this way, there is no room for interpretation or constructive discourse regarding the relevancy of monument, or the relationship between the form and the event that is to be remembered. Thus, this kind of representational monument essentially regards form to be little more than a less precise version of language, and hence places no value in the potential for meaning to form in the realm of experience. However, the changing attitudes towards remembering in the past century have 
affected a change in our approach to the public memorial. Many monuments today are not based upon a language of representation, rather, artists and architects have traded semiotic system for a more private autobiographical game of reflection and personal recollection.

An example of such a shift is Peter Eisenman's recently constructed Holocaust Memorial in Berlin, opened on May 10 2005, two days after the sixtieth anniversary of VE day. The monument consists of 2,711 concrete pillars, or 'stelae' planted close together to cover an entire city block. Some of the stelae are close to the

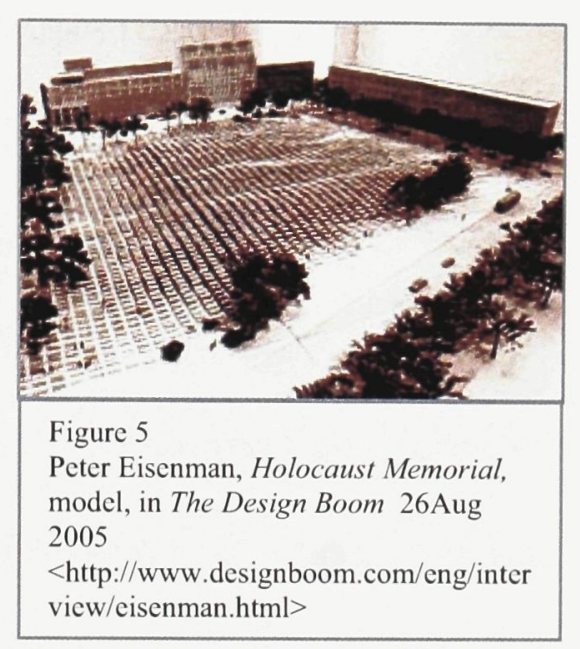
ground, and others stand upright to reach a height of 4.7 meters. This field of pillars is accessible from all four sides, and is open twenty-four hours a day, seven days a week, Eisenman hopes that this will contribute to the to integration of the memorial into the city. The monument is constructed on a historically significant site in Berlin, a 19,000 square foot piece of land that once housed the office of Nazi propagandist Joseph Goebbels, and is situated just south of the Brandenburg Gate, a gate constructed in 1791 to represent peace yet ironically incorporated into the Berlin wall. Nearby was also Hitler's Chancellery as well as the infamous bunker where he ended his life. After 1961, the site was adjacent to the Berlin wall and was part of the German Democratic Republic's (GDR) heavily monitored 'death strip.' 
But despite the number of historical events that occurred on and around the site, Peter Eisenman has made a conscious decision to de-contextualize the Holocaust, to remove any direct associations with the event itself. Instead, Eisenman's primary intent was to create privately moving experiences that allow each individual to form his or her own interpretations, relatively free from external rationality and nostalgia. Instead of representing aspects of the actual event by engraving names or images, Eisenman creates a blank field that people must make sense of on their own. With less than one meter between pillars, an individual will not be able to navigate side by side with a companion. Eisenman states: "I'm hoping that this monument will establish a different view of memory, a different view of monument, because it doesn't speak ... The city speaks: you look at that building, it speaks. Most graves have names on them, most memorials have names. This is an absolutely blank field ... The whole idea is, what does it feel like to be alone in space? What it is to be without any goal ... no beginning, no end, no direction? ${ }^{52}$ In essence, Peter Eisenman's memorial is a monument for remembering without signifying. Aside from the possible knowledge that the construction is in fact the Holocaust Memorial, you are left with only your own experiences to guide you through the field. Although those of us that did not experience the Holocaust will never fully comprehend its atrocities, Eisenman

\footnotetext{
${ }^{52}$ Peter Eisenman, quoted in Anonymous, "Freedom Site discussion: Architect on Berlin Holocaust Memorial," The Freedom Site Aug. 2005

$<$ http://www.freedomsite.org/pipermail/fs_discussion/2001-February/001442.html>
} 
attempts to create a memorable experience, a sympathetic condition between those participating in remembering and those that suffered.

The emphasis on participation is what separates Eisenman's approach from the traditional monument. Instead of sculpting likenesses or inscribing text that can be viewed at a glance, participants of the memorial must dedicate time to navigate the field. The period of time between when one enters and when one leaves the site is the extended time of encounter between a subject and the monument. As previously mentioned, the absence of signifiers provide no immediate indication of a proper engagement, and no clue as to what the Holocaust was. This indeterminacy can lead to a multiplicity of reactions. But even if one is unaware that the construction is actually a holocaust memorial, he or she may still be transported to the most remote corners of their own experiences when confronting the severity and unrelenting rhythm of the concrete forest. There is still the possibility that this encounter may bring about genuine states of trepidation, uncertainty and solitude. Yet, if one is aware that this is indeed the Holocaust memorial, he or she will more than likely use the encounter as a passageway into the lives of those affected by the Holocaust, by superimposing personal experience with one's own knowledge of those atrocities. Nicolai Ouroussoff, writer for the New York Times, gives the following account: "At first, you retain glimpses of the city. The rows of pillars frame a distant view of the Reichstag's skeletal glass dome. To the west, you can glimpse the canopy of trees in the Tiergarten. Then as you 


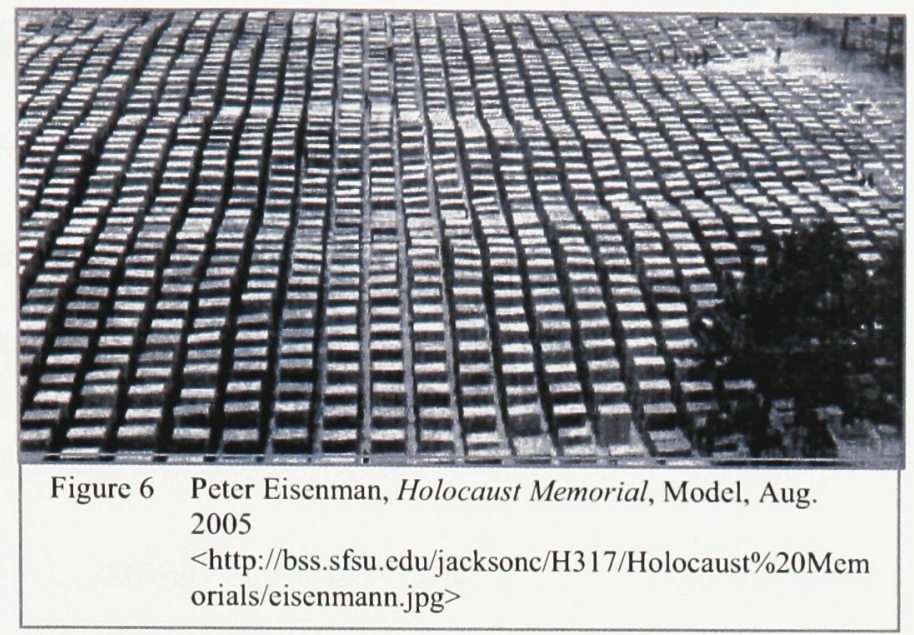

descend further, the views begin to disappear. The sound of gravel crunching under your feet gets more perceptible; the gray pillars, their towering forms tilting unsteadily, become more menacing and oppressive. The effect is intentionally disorienting. You are left alone with memories of life outside -- the cheerful child, for example, balanced on the concrete platform.

This is a chilling moment. For me, it evoked Primo Levi's description of the death camps. "To sink is the easiest of matters," he wrote in "Survival in Auschwitz." "It is enough to carry out all the orders one receives, to eat only the ration, to observe the discipline of the work and the camp." Only through constant struggle and arbitrary luck was survival possible." 53

Intrigued by the experience of the concrete forest, Ouroussoff ' $\mathrm{s}$ experience is an act of remembering the event in deeply personal way. His account of the

\footnotetext{
${ }^{53}$ Nicolai Ouroussoff,, "Architecture Review; A Forest of Pillars, Recalling the Unimaginable." The New York Times Aug. $2005<$ http://query.nytimes.com/gst/fullpage.html?res=9F01E6DF123
} 
experience is undeniably contingent upon personal experience. But along with these genuinely moving accounts, Eisenman has even accepted the possibility that some may find no significance in the form, even going so far as to use the surfaces for graffiti. When asked if the monument would be demeaned if antiSemitic comments were scratched on the surface, he answered: "Maybe it would. Maybe it wouldn't ... maybe it would add to it." ${ }^{54}$ Nevertheless, this possibility that the memorial will have little or no effect on an onlooker's understanding or sympathy towards the atrocities of the Holocaust, is the root of much public criticism of the project.

The indeterminate nature of the project is the reason behind its controversy. Critics such as Nancy Levinson of the online digest "ArtsJournal.com" questions the potency of an abstract Holocaust Memorial. Levinson believes that the main problem with the work is that onlookers will not be able to engage with the piece unless they have prior knowledge of the horrific event. Criticizing the observations made by Nicolai Ouroussoff, she writes the following:

"I couldn't help but wonder: to what extent does this sort of sensitive reading, this nuanced and detailed interpretation, require considerable prior knowledge? Ouroussoff tells us that he became disoriented as he wandered among the grid of closely spaced 
pillars, and that his unease evoked for him passages from Primo Levi's Survival in Auschwitz, passages that describe the despair and dehumanization of the camps. But what if a visitor to the memorial hasn't read Primo Levi's account of his ten months in a labor camp? Or indeed much of anything about the Holocaust? What if a visitor is among the fifty percent of Britons who - as the alarmingly informative web site of the History News Network tells us - have somehow never heard of Auschwitz? ... If you stumbled upon a five-acre field of 2,711 concrete pillars and didn't know that it was intended to be a "memorial to the murdered Jews of Europe," would it really communicate to you "the scope of the Holocaust's horrors"? ",55

Levinson's very expectation that any form can really 'communicate the scope of the Holocaust's horrors' is itself misplaced. Such a question is conditioned by the assumption that equates the built monument with representing fact and the expectation that all memorials should explicitly denote the facts and horrors of an event. But if these are the requirements of a memorial, there will be no further incentive to construct memorials. The expectation that a built structure can and

\footnotetext{
${ }^{54}$ Peter Eisenman, quoted in Anonymous, "Swastika Drawn on new War Memorial” Aug. 2005 $<$ http://msnbc.msn.com/id/7841104/>

${ }^{55}$ Nancy Levinson, "Pixel Points: In the Bubble," ArtsJournal.com Aug. 2005

$<$ http://www.artsjournal.com/pixelpoisnts/archives20050501.shtml $>$
} 
should communicate as lucidly a written fact sheet places every memorial ever constructed in question.

Although it may be true that a person with no prior knowledge of the event will not be able to connect their experience of the monument with the event itself, this same critique can also be applied to traditional memorials based on symbolism and representation. For example, the National War Memorial discussed above does depict soldiers as well as the dates of the war, but a person that knows nothing of these wars will not be able to connect with the actual events beyond the superficial recognition that it is some sort of monument to war. Similarly, a person that encounters a statue of a king from the fifteenth century with no previous knowledge of art history will not be able to decipher the symbols that adorn the statue, nor will they be able to relate the accomplishments of the man himself. Because no two people have had the exact same experiences in life, no two persons will have the same exact same understanding of the project. To refer back to Henri Bergson, the construction of a monument that only represents existing concepts essentially undermines our entire incentive to create form. In Levinson's case, form is built to arrest the event, but Bergson argues that this impossible by virtue of our ever-evolving human nature. The individual temporal exchange precedes our concepts, not the reverse. Nancy Levinson herself seems to sum up her own skepticism towards the communicative potential of tectonic experience, in her final statement that "it is not the vast grid but the wall text that 
supplies the memories." 56 If we were to place no value in the ability for architecture to mean beyond our already existing concepts, then we may as well use text in the place of form.

Ultimately, Nancy Levinson's comments included, this very discussion is the basis of value in an intervention such as Peter Eisenman's Holocaust Memorial. The rational grounding and the memorial itself become the catalysts for conceptual and sensual exchange. The validity of Eisenman's assertion, that de-contextualization will lead to potent personal experience, together with the actual personal experiences of the built structure opens the gates to the kind of aesthetic conversation that forms our understanding of the concrete. A unique response to monument such as this challenges us to reevaluate the significance of the event itself, to reconsider the holocaust as a tragedy that is still relevant to individuals in the world today, not as a mindless collection of facts related to a historical event. This is the strength of the weak monument that Sola-Morales emphasizes in his essay, it is the ability for a monument to trigger recollections at a personal level, to create an eventful memory that touches an element of the ineffable which is relevant to the contemporary subject. Collectively meaningful forms can only arise out of this kind of conscious reconciliation between how we perceive as individuals, and how we understand our world as a collective. In this way, architecture is not only the knowledge of form, it is an ever-changing

\footnotetext{
${ }^{56}$ Levinson, "Pixel Points: In the Bubble," ArtsJournal.com Aug. 2005

$<$ http://www.artsjournal.com/pixelpoisnts/archives20050501.shtml $>$
} 
philosophy of making, and ultimately, "to philosophize... is to invert the habitual direction of the work of thought." ${ }^{, 57}$

${ }^{57}$ Bergson, An Introduction to Metaphysics, 52. 


\subsection{Project: An Investigation in Five Acts}

By way of investigating the concept of weak architecture, this project uses as a procedural grounding the aspects of memory and self-conscious reflection in the aesthetic realm; more specifically, the idea that built form cannot induce definitive experience, but that potent experience can only emerge out of a selfreflective encounter as a product of singular interruptions of habitual thought. this work itself expands upon a temporal act: a collaborative effort to create a dance production. This experience itself then becomes a grounding for the design project that follows. This section will describe the evolution of the project. 


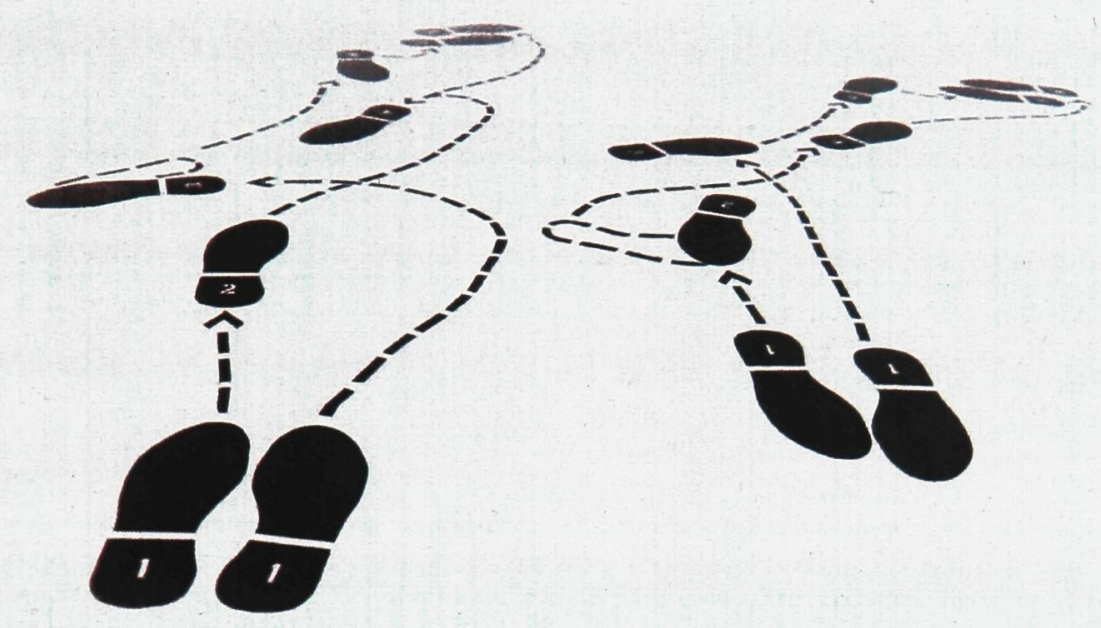

Figure 7

Dance Steps installed in main street of the School of Architecture

\subsection{Act One: Dance Steps}

The first act was performed with the intention of causing an interruption of the

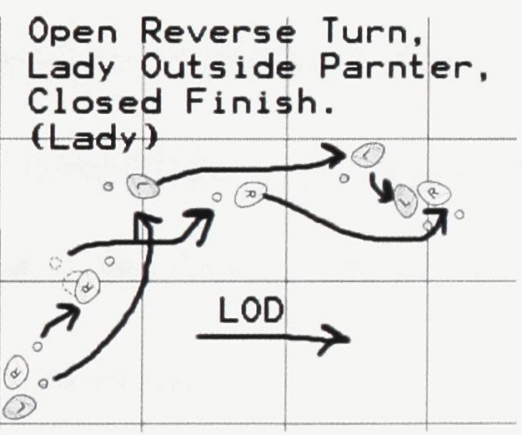

Figure 8

Basic left turn, Tango, Oct. $2004<$

http://www.umich.edu/ umbdc/DanceSyll abus/haagr/am_tango/am_tango_bronze/a mtg_leftturn_lady.htm>

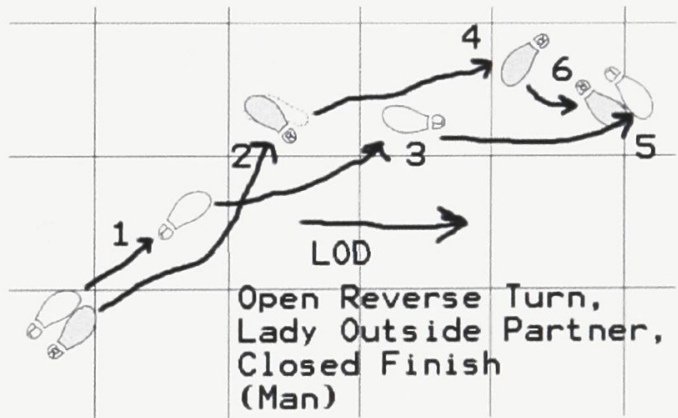

Figure 9

Basic left turn, Tango, Oct. $2004<$

http://www.umich.edu/ umbdc/DanceSyllabus/haagr/am tango/am tango bronze/amtg leftturn man.htm>

ordinary in order to stimulate a response and monitor reactions/engagements. An instructional diagram that outlined the sequence of the tango step was pasted (1:1 
scale) on the concrete floor of the main 'street' in the School of Architecture, an area of both high pedestrian traffic and communal gathering. In the diagram, the lead and the follower's steps were thrown askew and all steps were rendered with the same shoe print. Arrow and numbers indicated the order of the steps, but not how the two dancers should interact. A video camera was set up in a partiallyconcealed location overlooking the area in order to document the reactions of people to the installation.

The reactions ranged from a passing glance to a partnered engagement with the steps in an attempt to decipher their meaning. Several bystanders even managed to interpret the steps beyond their explicit instruction, realizing that they were in fact tango steps that depicted the point during which the dance when the lead dips his or her partner. By the end of the day the steps were more or less accepted as part of the landscape, and garnered less attention than they had in the beginning. Out of all possible reactions, the most unexpected results were the self-conscious realizations that activity was being recorded from the office above. These realizations were quick deterrents for activity. A passerby was less inclined to participate upon realizing that the steps had been put into place to monitor response.

The act itself is not architectural per se, yet it is useful in illustrating the relationship between architecture and the event at the most basic level. Although largely unintentional, the unassuming dance notation, together with the video camera in the director's office draws an explicit viewer-viewed connection 
between the two spaces. Even if the relationship between architectural elements has always been present, the event ties them together in the personal encounter. One can only assume that a person walking through the street will be increasingly aware of the eyes that may be watching from above, even if the effects are only temporary. Of course, after the recording ceases, only the steps remain to pass into the realm of the ordinary.

These simple connections between spaces constitute the base for architectural experience. Even after a person has walked through a building numerous times, the building can still speak through the events that occur within its walls. Although these events are impossible to predict, architectural meanings are assembled anew, contingent upon personal memory and experience.

By accepting that architectural experience can neither be represented by form, nor induced by it completely, the weak link between the architectural idea and the architectural experience is the territory for architectural exploration. The event of creating is inevitably separate from the event of experiencing by virtue of the fact that the experience has not yet occurred. 

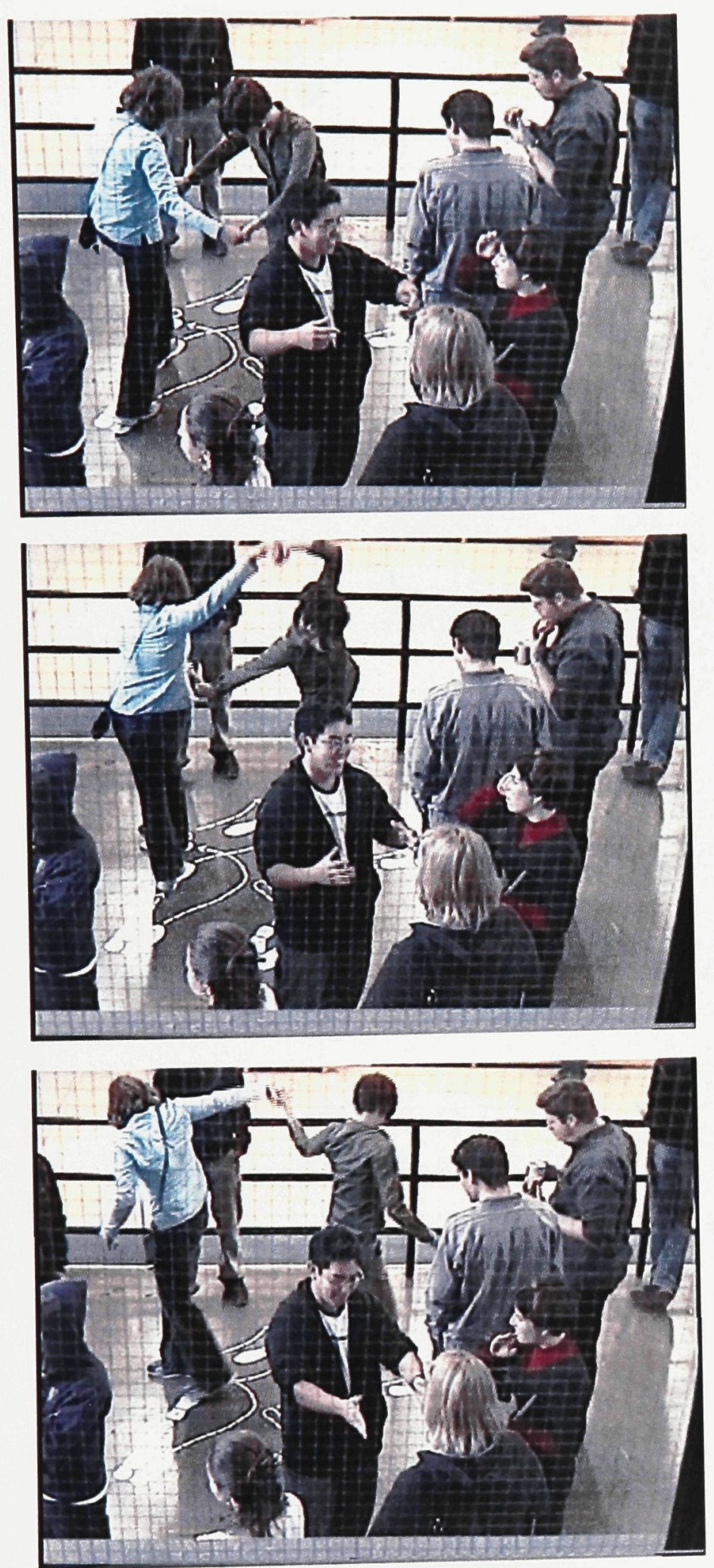

Figure 10 Still sequences from recorded footage of the dance steps

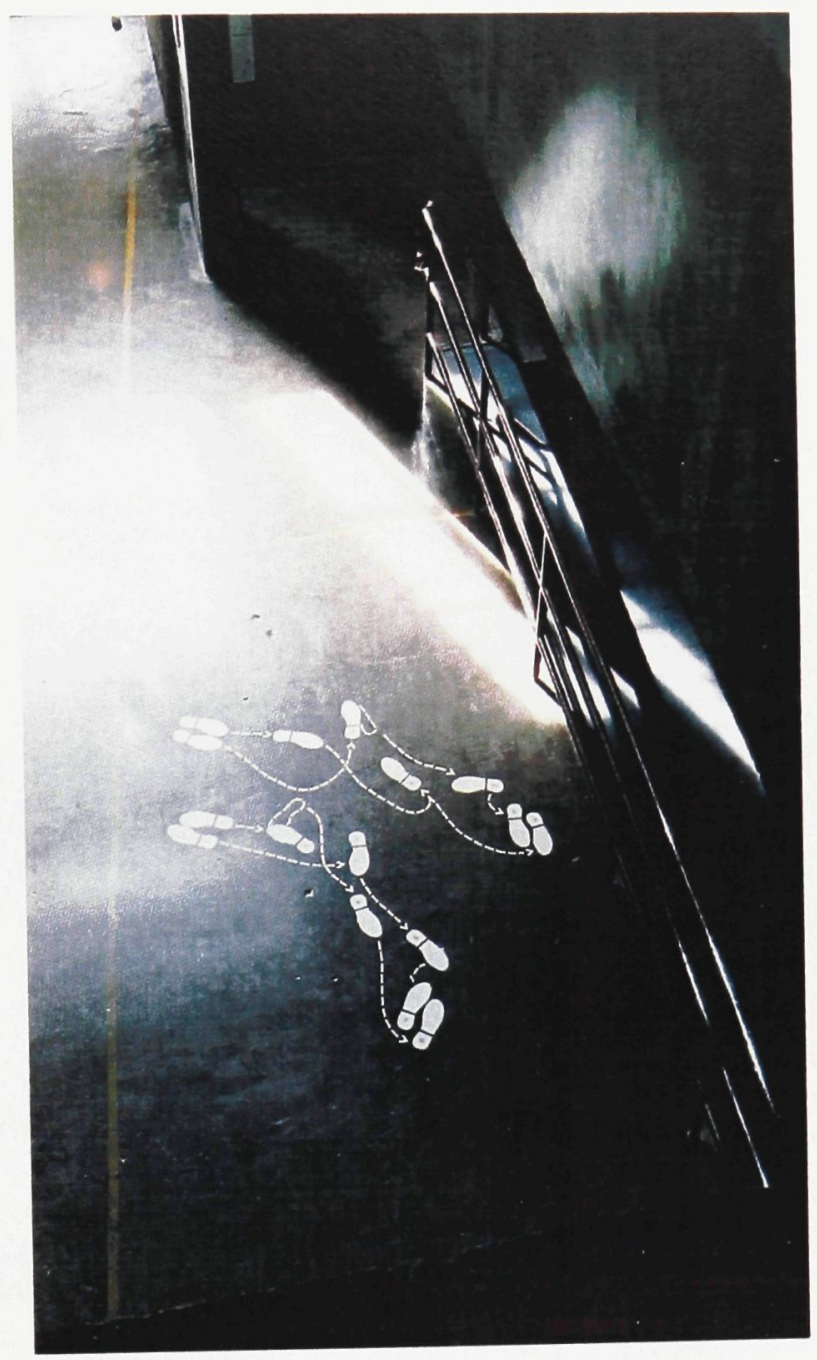

Figure 11

Dance Steps

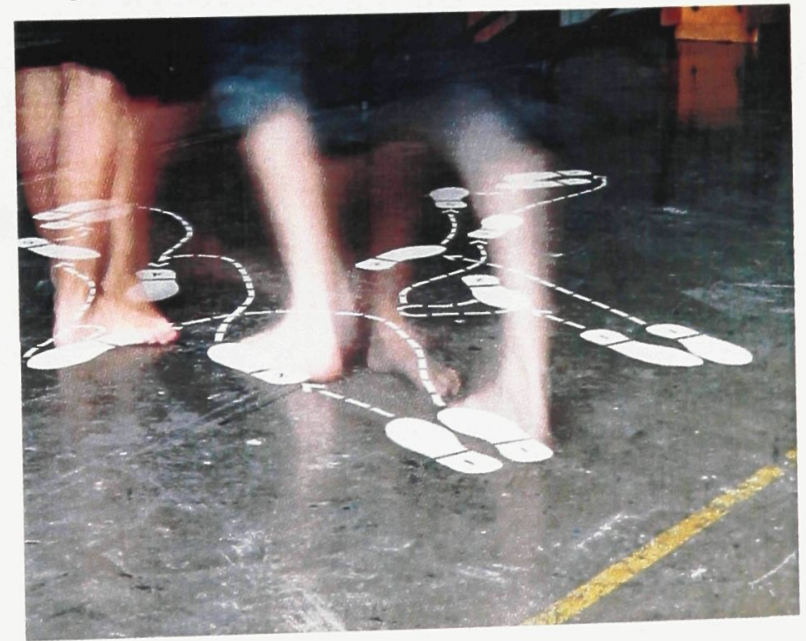

Figure 12 Detail of Dance Steps 


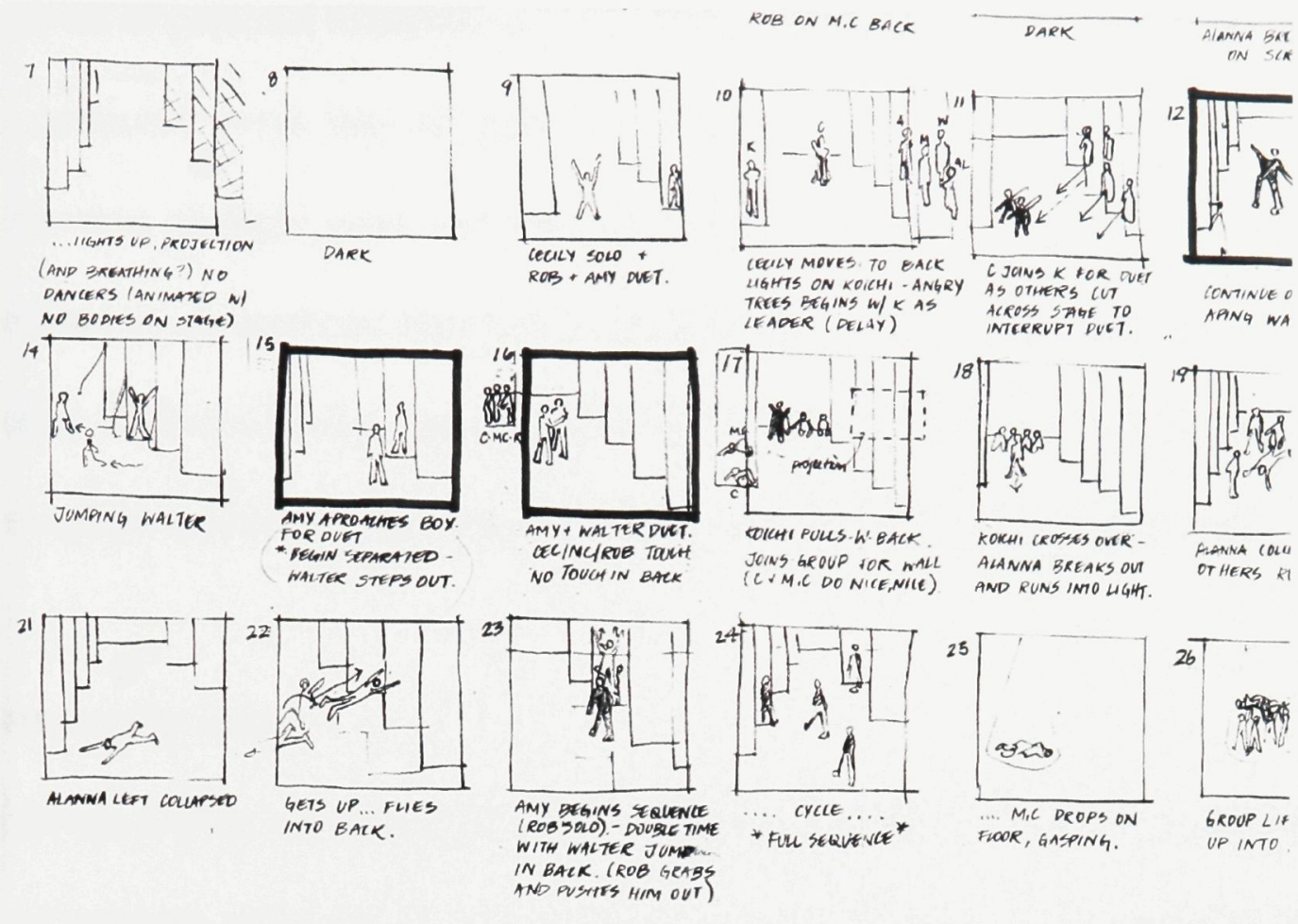

Figure 13

Detail of the story board

\subsection{Act Two: An Interdisciplinary Collaboration}

The second act was carried out as a three-week process with Le Groupe Dance

Lab in Ottawa. It was a collaborative process that united choreographers, dancers, architects, a videographer, a composer, a lighting designer and a set designer in the creation of a single performance. The event stands alone as an exploration in dance and spatiality initiated by the creative director of the lab Peter Boneham. However, my intent for integrating this process into this particular architectural exploration is not entirely separate from that of the lab. The nature of a collaborative event is such that individuals from different disciplines are brought together to take part in the same process. In this way, each individual stems from a separate theoretical grounding, in addition to having a 
unique set of personal experiences. This kind of theatrical creation can only occur as a temporal event that all parties engage in. It is a creative process that incorporates multiple goals and multiple groundings into a singular performance to be read as an aesthetic experience, demonstrating that we are able to converse on many different levels, proving that discontinuous theories do not translate into meaningless creations. This section will describe the process in greater detail.

\section{Notes on the Lab}

Le Groupe Dance Lab stemmed from a desire to mine the wealth of ideas locked within creative intention by encouraging experimentation. In Peter Boneham's own words," the Lab rose from the need for a major eruption in the dance community, an eruption that would cause the orchestration of a startling new level in creative investigation." When Peter Boneham created Le Groupe Dance Lab in 1988, he fashioned a new typology for dance, one that defines the dance studio as laboratory, the choreographer as inventor, and ultimately dance as the alchemy of human motion. This focus on discovery in the creative process is unique to Le Groupe; other dance companies typically devote their resources to refining a single production, and are therefore not committed to pushing the boundaries of the experimental stage. According to the Dance Lab: "Seasoned and emerging choreographers are invited to apply for a residency with a focus on the creative process, not production. The Lab sessions concentrate on the process of making dance, without the stress of delivering a full-production work at the end. 
Choreographers come to the Lab with a specific goal in mind: to find new sources of material, to explore their movement vocabulary, to experiment with a complementary art form, to further investigate their creative process." Not intended to replace dance production companies, the Dance Lab is an invaluable resource for choreographers that are intrigued by possibility, and choose to pursue an investigation into their own process of creative discovery.

A typical season in the Dance Lab is structured with nine residencies. Each residency is headlined by a visiting choreographer, and a monitor or critical outside eye that gives the choreographers input into their process. Essentially, the lab provides the choreographers with a troupe of exceptional dancers, production coordinators, studio and theatre space, publicity and media coverage, as well as the opportunity to perform to a live audience for feedback. Choreographers that successfully apply to the lab have these resources at their disposal for a three to four week period, the product of which will be performed for an audience. The work, when performed, is not meant to be seen as a finished piece, rather as a work in progress that may or may not eventually lead to a finished productions. The lab can be seen to work on many levels: first is the obvious impact that a residency can have on the work of a participating choreographer. By giving choreographers the freedom and resources to experiment, future projects can be shaped by material explored during the lab sessions, often maturing into full length productions. Second, the choreographic research conducted by each resident contributes to an overall understanding of dance and choreography that is 
invaluable to the dancers and the monitors of the lab. The dancers themselves have the opportunity to work with countless choreographers in compressed timelines, hence they learn skills in improvisation and become very adaptable to various modes of operating. Meanwhile, observers of the lab can also be inspired

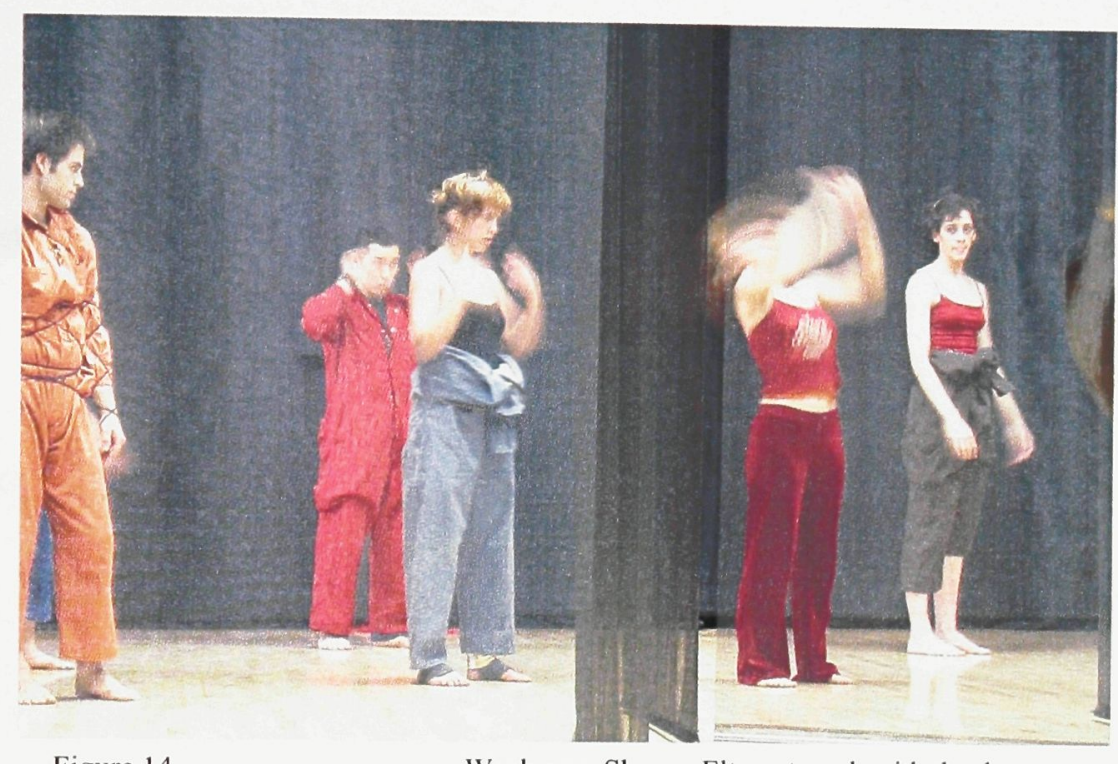

Figure 14

Week one: Shauna Elton at work with the dancers

by the ideas explored in the lab sessions. Finally, the lab provides outsiders of the industry with a unique opportunity to engage in choreographic discourse. The discussions that follow each performance not only allow choreographers to obtain feedback on their work and their intentions, they generate a mutually beneficial culture of discourse between dancers and viewers within the population. With a better understanding of the vocabulary of dance, viewers are more likely to be intrigued by dance. Ultimately, Le Groupe Dance Lab acts as a catalyst for innovation, pushing the boundaries of dance by testing the unknown in an environment of absolute veracity. 


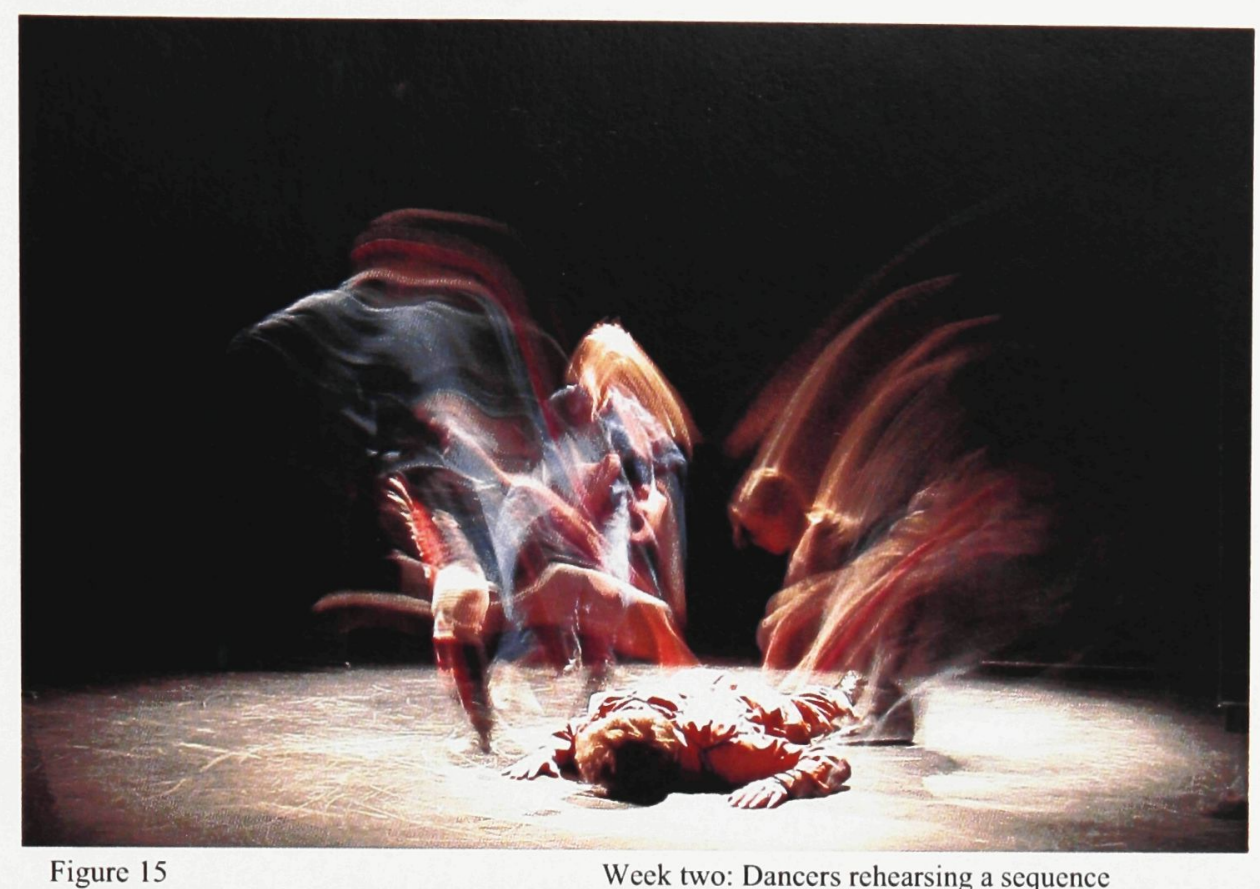

\section{Fast Track Process (October 2004)}

The Fast Track process was a special event fundraiser developed by Peter Boneham to collaborate with outsiders of the dance community.

The list of participants are as follows:

Monitors Loretta Kong and Derek Fleming

Choreographer no. 1 Shauna Elton

Choreographer no. 2 Robert Abubo

Set Designer $\quad$ Normand Vandal

Lighting Designer Paul Auclair

Composer Olivier Girouard

Videographer Matthew Cameron

Dancers Robert Abubo

Marie Claire Forté 


\title{
Cecily Greenfeld
}

\author{
Alanna Kraaijeveld \\ Walter Kubanek
}

Amy Schindel

Koichi Yano

\section{FastTrack/ Weak Intentions}

A strong overriding intention for the whole process is rendered impossible by virtue of its structure. The only singular intention for the piece came from Peter Boneham himself, rooted in investigating the differences between an architect's spatial understanding and that of a dancer. Furthermore, it would be counterproductive to even attempt to contain a process-oriented collaboration within a singular premeditated framework. Each of the fifteen people involved necessarily contributes to both the process and product. Hence, the performance itself is free to become whatever it is in the bodies and minds of the participants, and ultimately that the audience.

The intention of this process is to generate discourse by assigning two architecturally trained monitors to assemble a selection of non-cohesive dance material into a performance. Typically, the role of the monitor is to act a an outside eye to the process and to provide critique and guidance for the choreographer during a lab residency. They offer a different perspective on dance material a step removed from the source. Although they are outsiders to the 


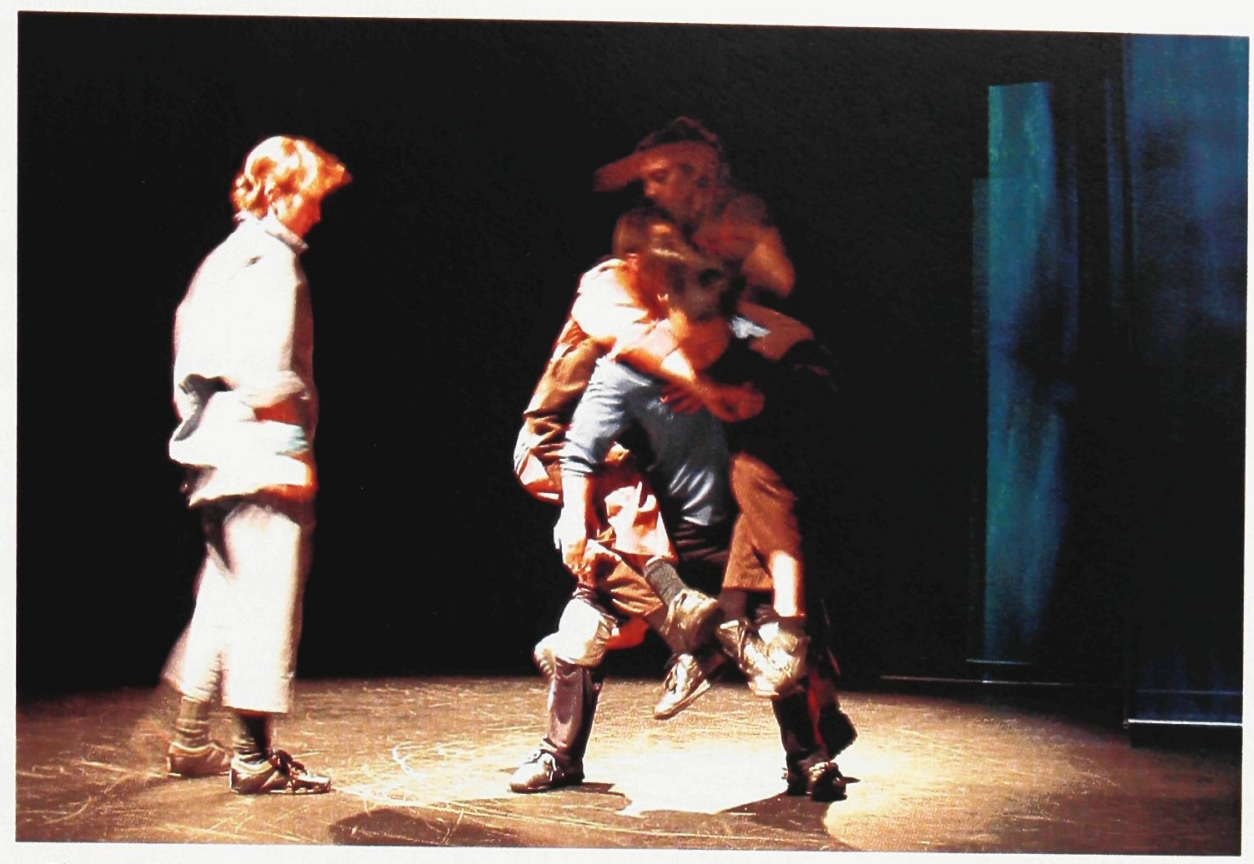

Figure 16

Week two: Dancers climb on Koichi Yano

process, monitors are usually insiders to dance. Often this responsibility belongs to the artistic director and founder of the Lab, Peter Boneham. In a typical process, the monitor is not responsible for anything more than offering input; it is the choreographer's own responsibility to assemble the pieces for the audience viewing. In this particular process, Derek Fleming and myself served as modified monitors, responsible for conceiving and assembling a full performance from fragments of dance material, created by two choreographers. As part of this task, the monitors also orchestrated factors such as the use of the set, lighting, video and sound. Essentially, the timeline of this process can be broken down into three stages that took place over three weeks. During week one, the first choreographer was responsible for developing a dance vocabulary inspired by a stage set already designed by the in-house designer. During week two, the second choreographer 
develops an independent set of dance sequences for the same setting. During week three, the movement vocabularies of both choreographers are spliced together on stage by the monitors along with sound, lighting, and video, all to be performed at the end of the week.

\section{Reflections on processes}

Architects and dancers are both creators of space. As mentioned before, choreographers and dancers function uniquely within the temporal event, hence the dance is developed within the medium itself, and not its representation in the form of text or image. In contrast, architects more often than not, create solely within representational mediums. The process of creating modern dance is aligned with many of Ignasi de Sola-Morales and Henri Bergson's concepts. It is the act of creating meaning through the medium of the human body, and is thus absolutely inseparable from the temporal moment. Furthermore, unlike more structured forms of dance such as ballet, the goal of modern dance is precisely to decipher the communicative potential of the human body beyond representation and tradition. Ottawa's Le Groupe Dance Lab is devoted entirely to experimenting with the creative process of choreography.

In this particular process, both choreographers were free to create sequences that were initially free from a guiding concept or idea. Instead of being responsible for a finished performance, Shauna Elton and Robert Abubo were each given a week to develop sequences that react to a particular stage set. 
Hence, the role of the choreographers in this case was not to create dance phrases with the intention of assembling them into a finished form, but rather to react to an environment, and therefore create gestures that could be reinterpreted later by another artist. In this way, the choreographers were not responsible for stepping back to reevaluate what they had done as a total composition; instead they could remain in the moment of the dance stage itself, to develop gestures with limited pretension towards the totality of the performance.

Because the creation of dance itself occurs as a temporal event, dance is inseparable from the dancer's bodies. Ultimately, dancers must operate within the moment of duration. This is evident even in their treatment of conceptual ideas. For the first choreographer Shauna Elton, words were not a generator for the dance as much as they were a device for remembering themes within bodily phrases. The word 'polarity' was used as an initial generator for action, but was neither the fixed concept for the dance, nor a point of departure for all sequences. Titles such as 'angry trees' and 'free fish' were also attached to each sequence as a method for remembering. The sequences developed by Robert Abubo were even less conceptual. Robert's process was almost entirely focused on experimenting with the intuitive reactions of dancers themselves. For instance, to create one particular sequence, Walter Kubanek was asked to sit down and watch the other dancers perform their sequences; immediately after the last dancer had finished, he was told to act out all that he remembered from what he had seen. The result was a sequence constructed of fragments from all the other sequences. In this 
way, the dance sequences were developed from a variety of factors contingent more upon intuitively exploring body in space rather than representing concepts.

As monitors, we were given a selection of sequences developed around the same stage set with many small themes, but no single narrative. In effect, we were to act as interpreters of meaning, assembling movements and gestures based upon our own biases. Instead of crafting movement, we placed it in space and time. The process of converting dance material into a performance is essentially an aesthetic exercise that involves first interpreting the movements from the outside, then reassembling them as found objects. It is understood that the movements are themselves a medium of exchange that have no ideological counterparts, that the meaning for each gesture is layered and multiplicitous, contingent upon many different factors.

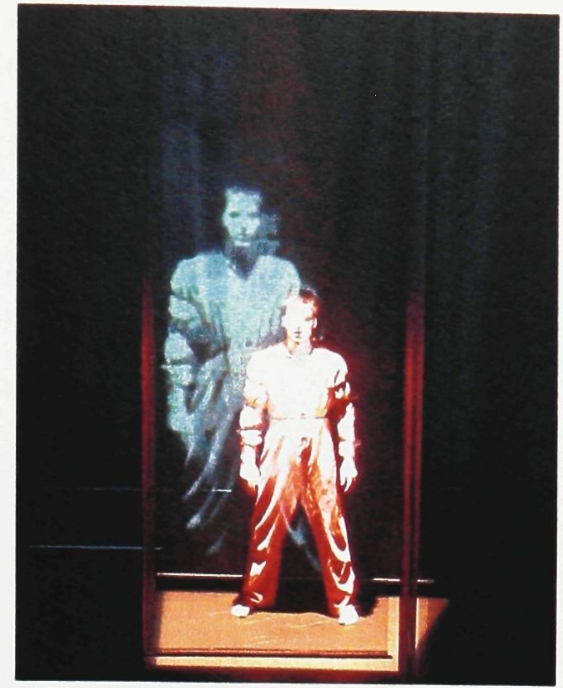

Figure 16

Walter Kubanek

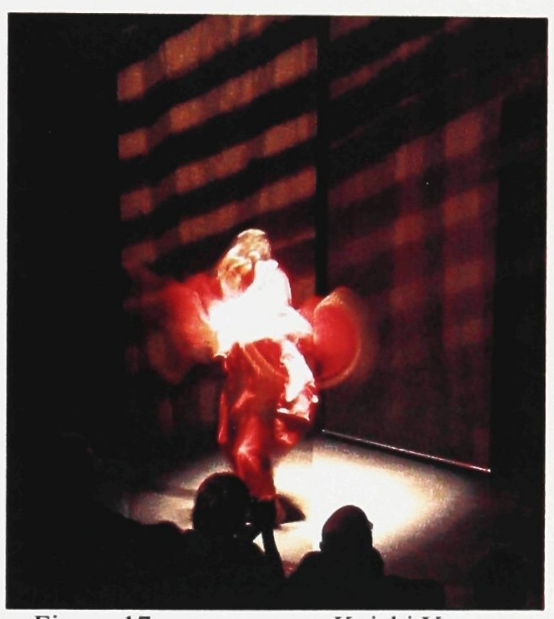

Figure 17

Koichi Yano

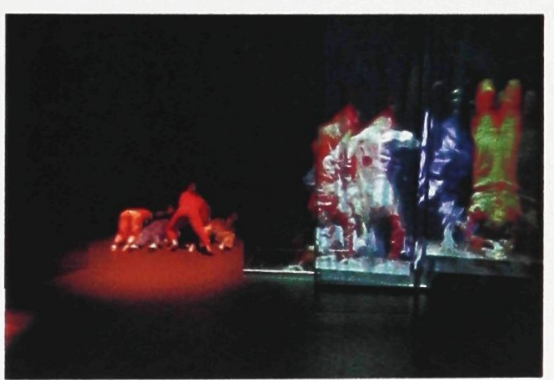

Figure 18 group wall sequence

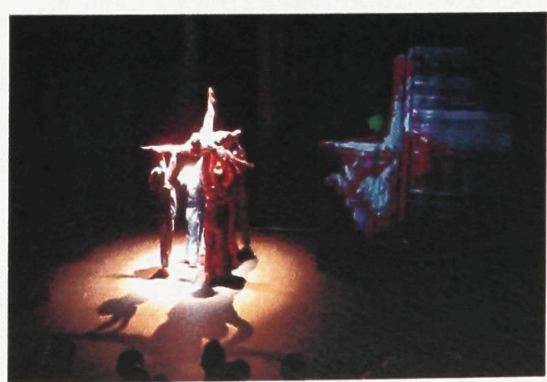

Figure 19 Marie-Claire forte is lifted 
With this in mind, our outside reading of the material was coupled with the self-conscious realization that this was not to be a narrative based performance that could be read in any one way. Therefore our intentions for organizing the

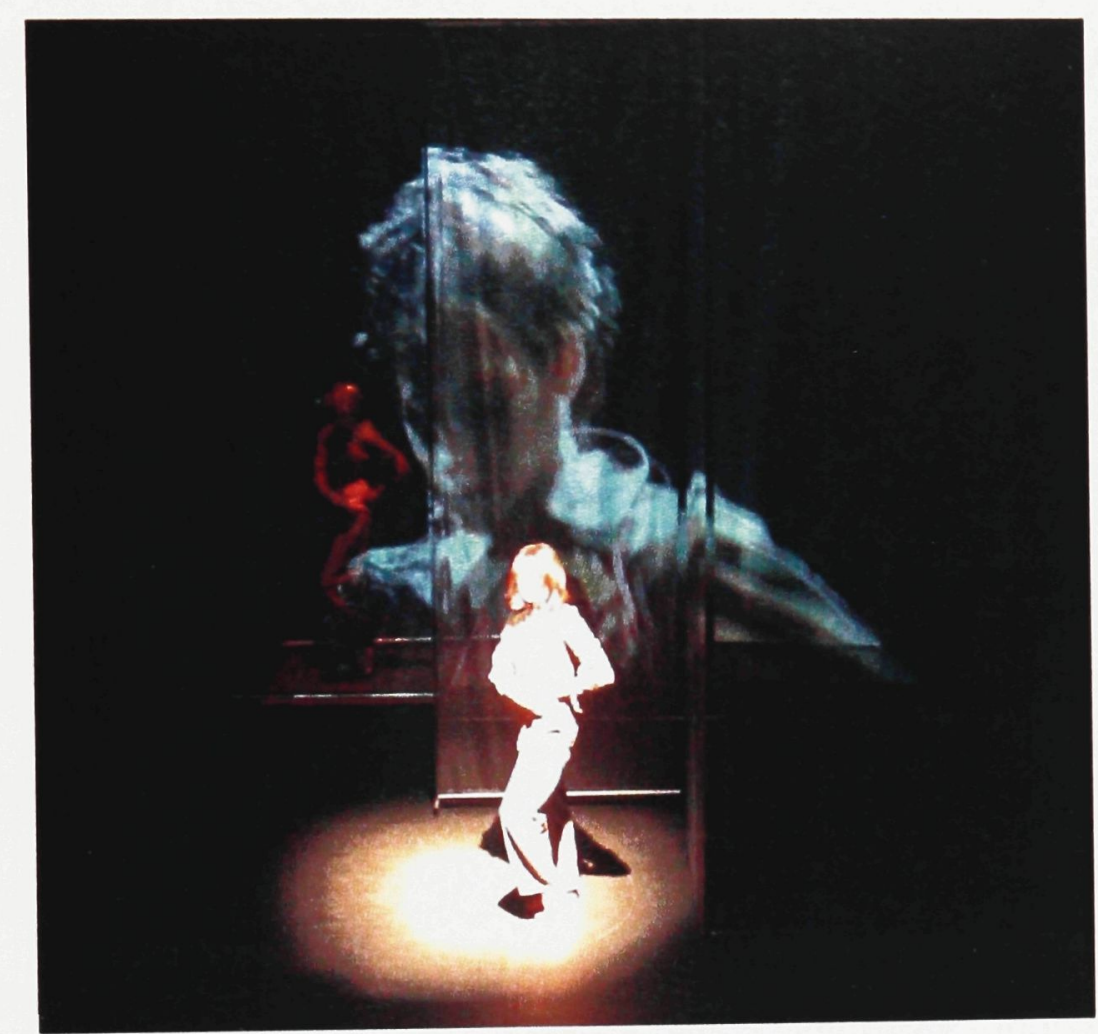

Figure 20

Cecily Greenfield and Marie-Claire Forte perform the final sequence

performance were directed at implicating self-conscious readings from the multitude of viewers. Emphasis was placed not upon explicating an interpretation, but upon teasing the space of memory and self-conscious reflection within the duration of the performance itself. For instance, video projection was one medium with which temporal overlap could be suggested to an onlooker. All video footage was taken from the performance itself, so that the projections during the show were always a depiction of the dance taking place. The moment 
of overlap occurs within the ambiguity of the source. Instead of using exclusively live or exclusively recorded footage, both were incorporated into the performance. Thus, it does not become clear which projection is live and which pre-recorded unless the viewer views it with the intent of finding out. At times the projection appears to refer to a central character on stage, but in fact depicts a character that is out of plain view, a revelation that is not immediately apparent to the onlooker.

The theme of memory and recurrence also emerged as an organizing principle for the overall structure of the dance sequences. Because the dance phrases were combined results from two different choreographic sensibilities, the integration of both onto one stage allowed for adjacencies had potential to affect the readings of the piece. The layering of these adjacent readings were explored within the duration of the dance. Fragments of sequences appear and re-appear out of the original context. These recurring sequences refer an audience member back to a previous points in the performance. Like the revelation of life and prerecorded footage, the assemblage was ultimately an attempt to weave personal recollection in with immediate observation through soft implications, and effort to generate more intimate, personalized readings of the work

In many ways, the process of creating dance helps to illustrate the relationship between the intuitive act and rational thought. As a literal act of creating in the medium of bodily movement, modern dance cannot be expressed through latent concepts, and cannot exist without the human body. The entire existence of modern dance is contingent upon the ability for the aesthetics of body 
movement to convey knowledge in a way that our pre-existing rational concepts cannot. Conversely, the project itself will cease to be relevant to any of the participants if it were not for the initial intent of the investigation. That is, if it were not for the intent to bring architect and choreographer together in a mutual investigation of space, the project would cease to be a constructive endeavor. In this way, the initial intent produces grounding for an act that then triggers selfreflection, and so on, an example of the perpetually expansive cycle of invention discussed by Henri Bergson. 


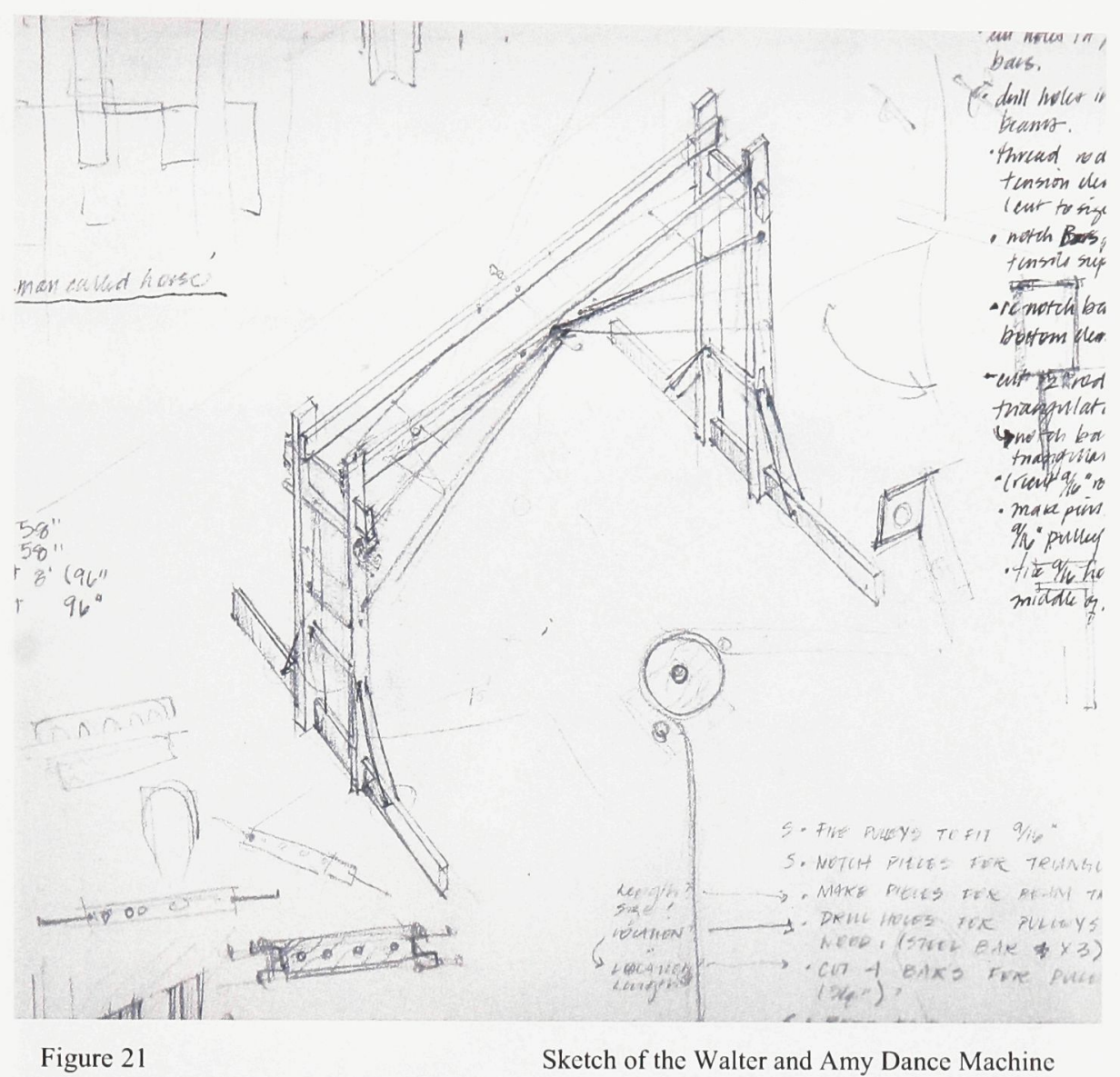

\subsection{Act Three: The Dance Machines}

Although the performance itself has been thoroughly documented from various angles in video and still image, the event itself will never be replicated. This next act attempts to expand upon the event by building upon its memory in a form that can be experienced in the temporal realm. Instead of representing the dance solely through image or video, these constructions are attempts to memorialize the dance in action. In this way, they are always conscious of the fact that they will be interpreted as experiences, not representations of past events. Hence, these machines can only be engaged with at a one to one scale in a personal visceral 
exchange. The intent of these constructions is to translate the actual event into built form, without resorting to manipulating its image.

Construction One: Walter and Amy Machine

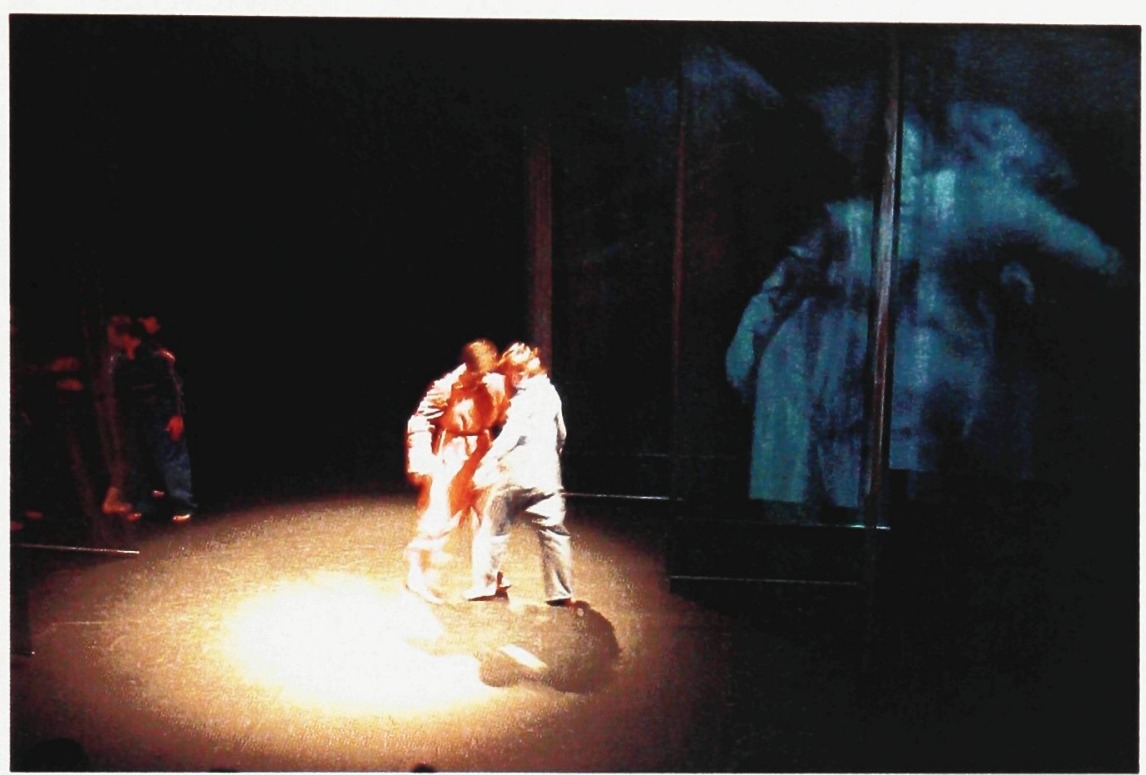

Figure 22

Walter and Amy in source performance for the dance for the construction one

The source performance of the first construction is a short duet between Walter Kubanek and Amy Schindel, in which the two dancers continually reach for each other but never engage in contact. Like polar opposites, they can try to touch each other, but an unseen force continues to push them apart. For instance, each time Walter reaches out to touch Amy, he is met with an equal and opposite reaction that pushes her even further away. The dance is performed very intimately, with bodies in close proximity to each other. Instead of attempting to represent this duet in its entirety, the intent of the construction is to isolate the simple action of reaching for another body without possibility of contact. Instead 
of formalizing the actual duet as it was performed, this machine attempts to frame the act, to expand the memory of the act into current experience as opposed to freezing it as an expired event.

The construction itself is a frame that surrounds a space approximately three meters cubed. Within the frame is a network of pulleys that links together two bodies so that motion in one will reflected in an equal and opposite motion in the other. More specifically, with the two subjects standing face to face, the frame connects hands, feet, waist and head together into a single system, so that the movement of one subject necessarily affects the other. Aside from binding two bodies into one system, the subjects are free to interact with each other as they please. In this way, the structure is free to move beyond the original event to become an experience in the temporal realm. 


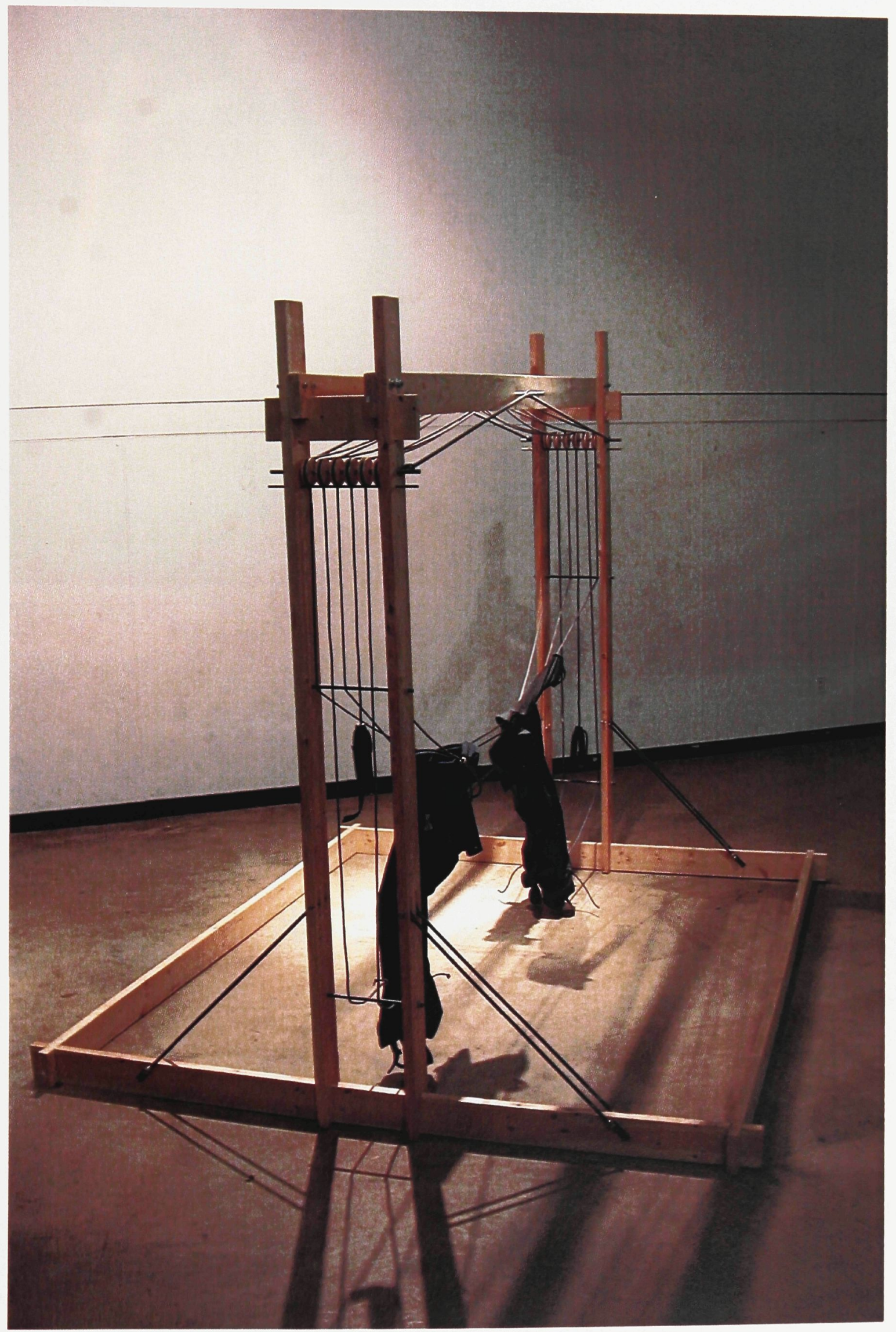




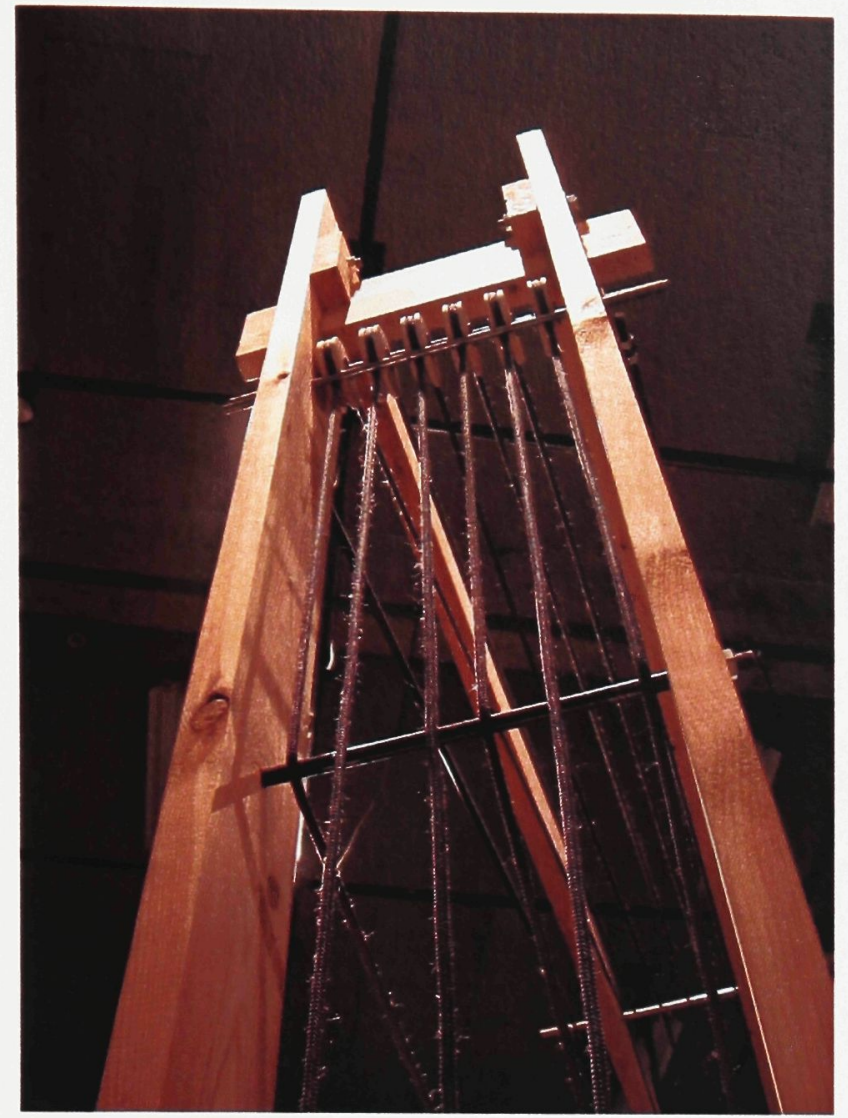

Figure 24

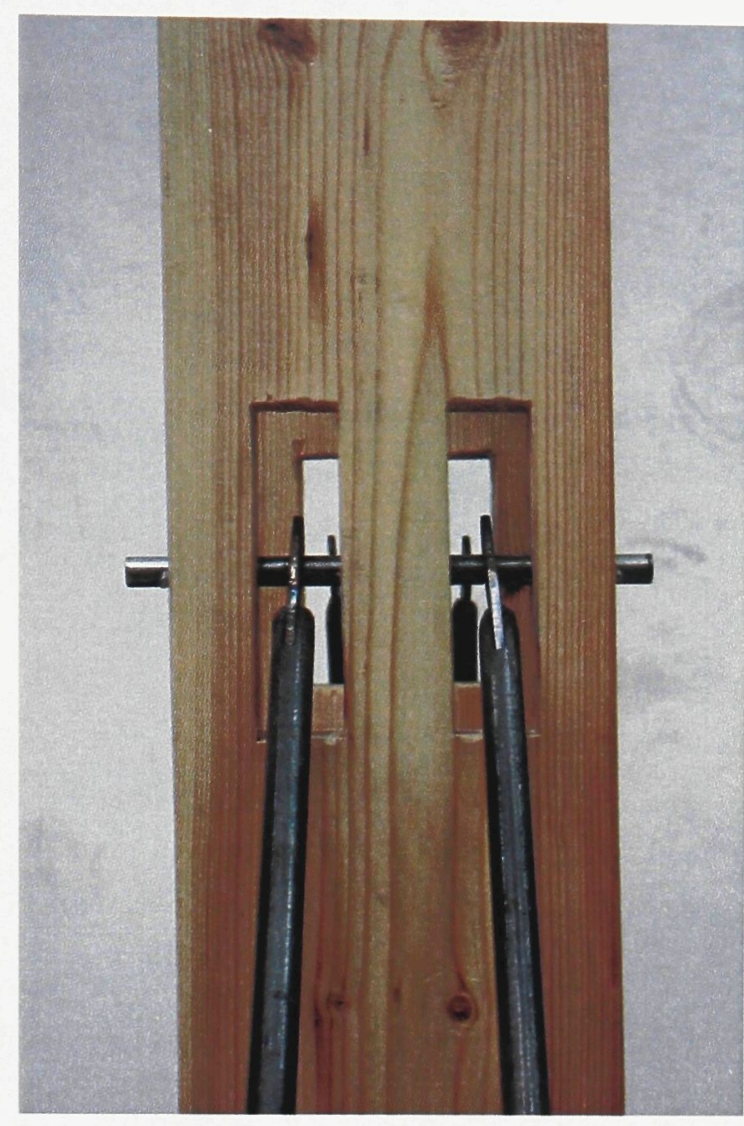

Figure 25

Support System

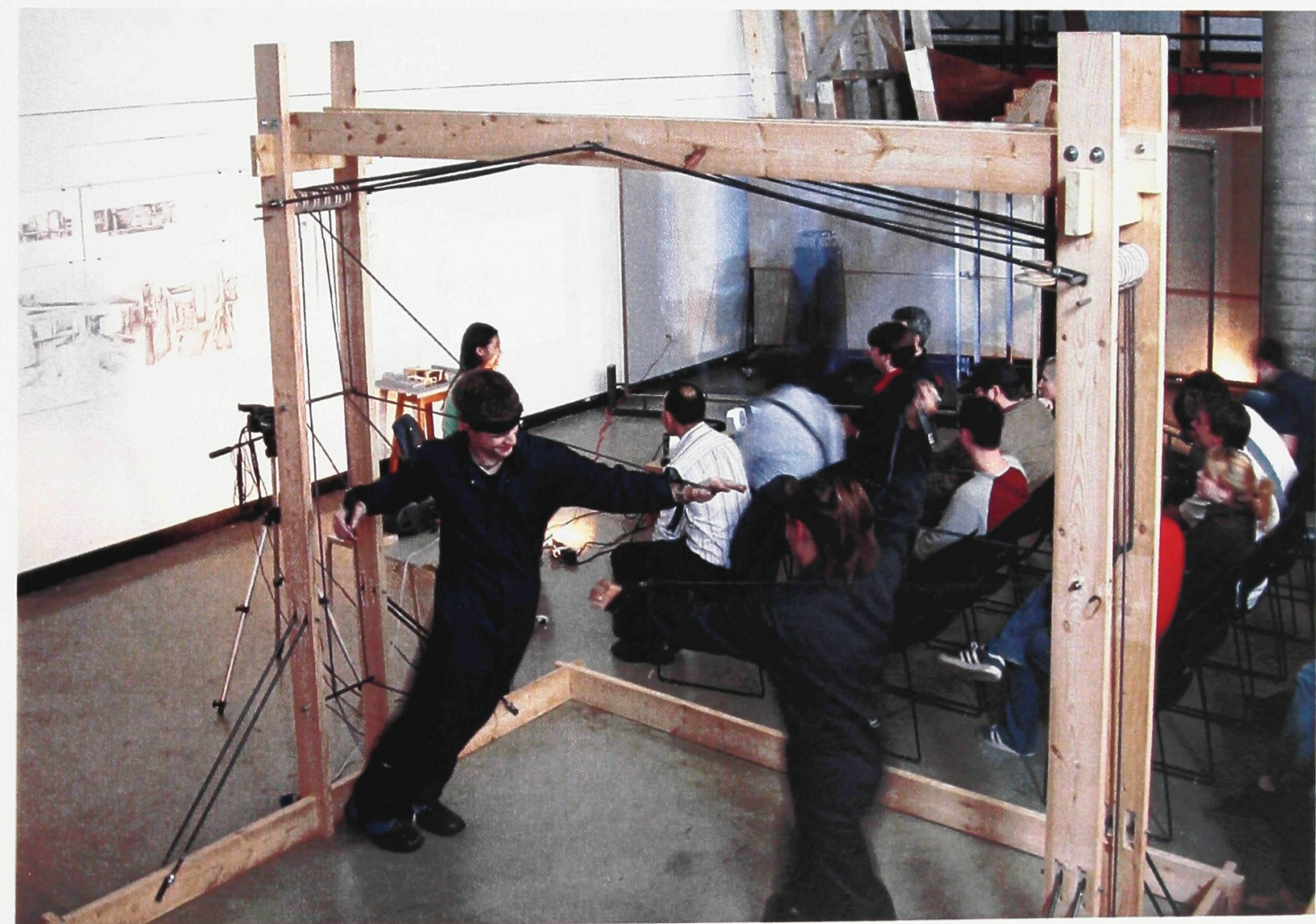

Figure 26

'Walter and Amy' in Operation 


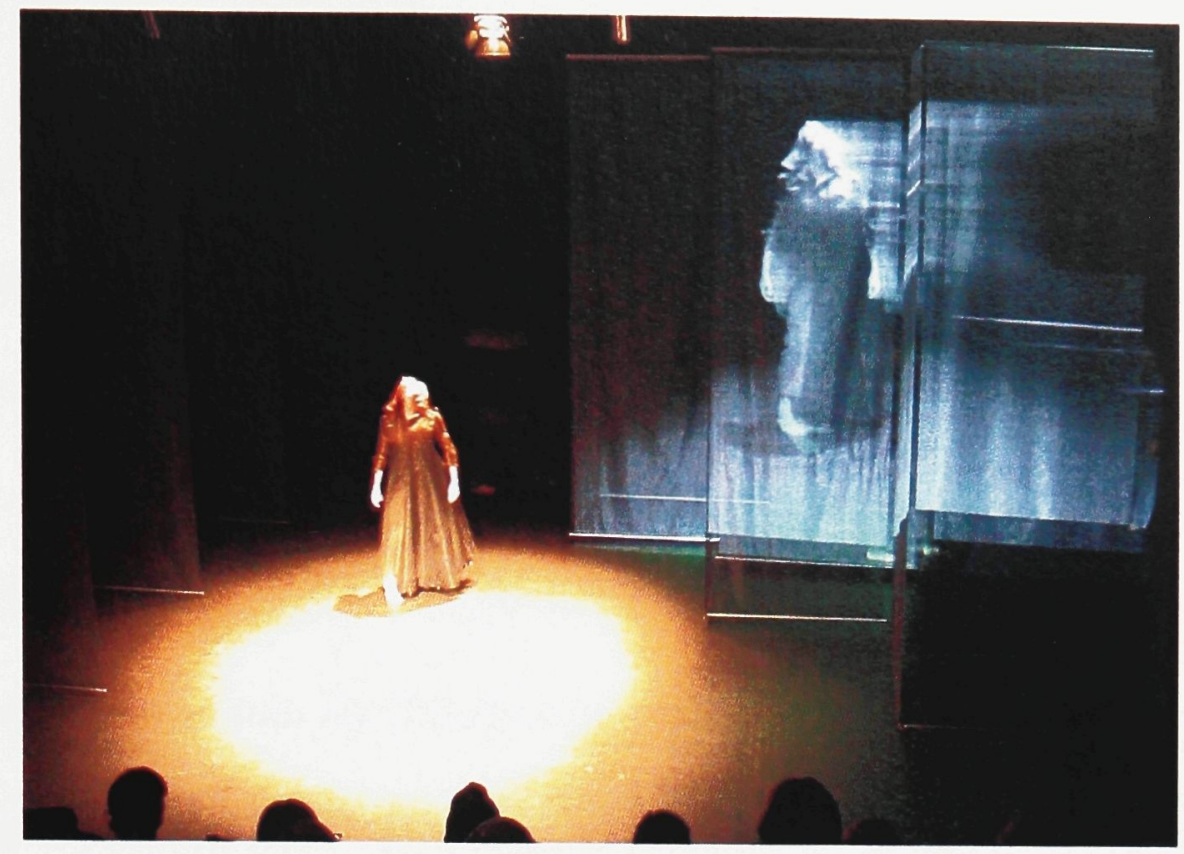

Figure 27

Cecily Greenfield's solo

Construction Two: The Dress

The source of the second construction is more complex than that of the first. This machine is based upon a sequence with more layers of interpretation than the Walter and Amy duet. The source of this construction is a solo performed by Cecily Greenfield in which she wears a dress that is constructed with the same material as the screens on stage. Weights are fixed onto the bottom of this dress so that they create a unique sound each time she touches down upon the ground. While she performs this solo, live footage of her performance is projected on the screens behind her. This solo is performed again at later point in the show, except that Cecily is no longer wearing the dress that she had at the beginning. The projection on the screen behind her refers back to the earlier performance, and depicts her performing the solo with the dress. Halfway through this second performance, she herself becomes conscious of the projection and begins to 
mimic her own actions, she promptly leaves the stage following this awareness, leaving only her projected self to complete the sequence.

Instead o. But instead of wearing a dress, a subject interacts with a series of pulleys that affect the movement of a dress two meters away. Depending on which pulley one chooses to interact with, the dress will spin at different rates. Layered upon this action are screens that separate the subject's body from the dress itself. So a participant would have to watch the dress spin, to some degree through a screen. In this way, engagement with the dress is mediated by the machine. Instead of simply engaging with a dress that is worn upon one's own body, movements are reflected in a dress two meters away and physically separated by a light screen, so that it acts as an imperfect reflection of the subject's own body movements. Like Cecily's solo performances, this machine is intended to layer action with recollection of previous action. 


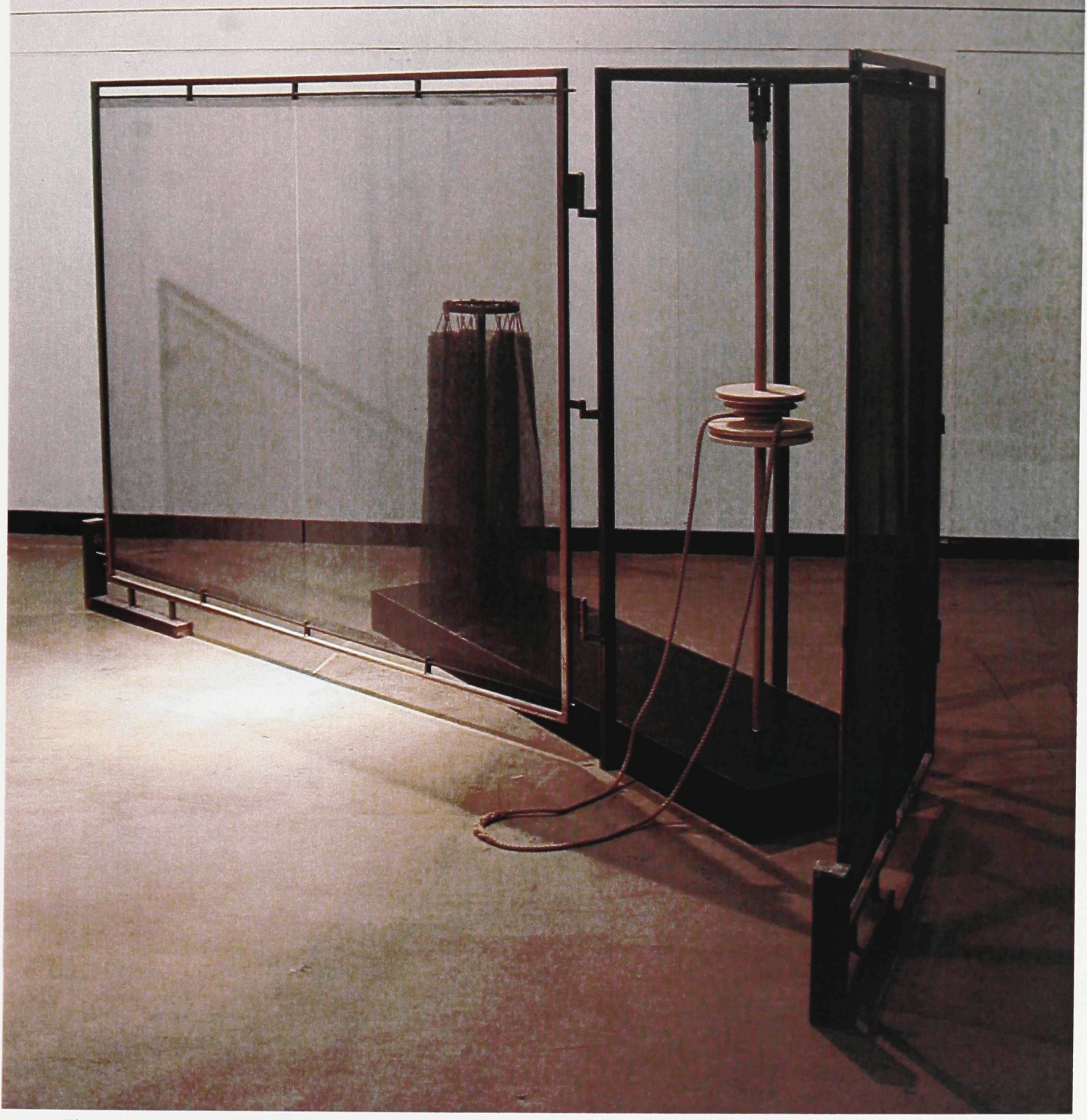




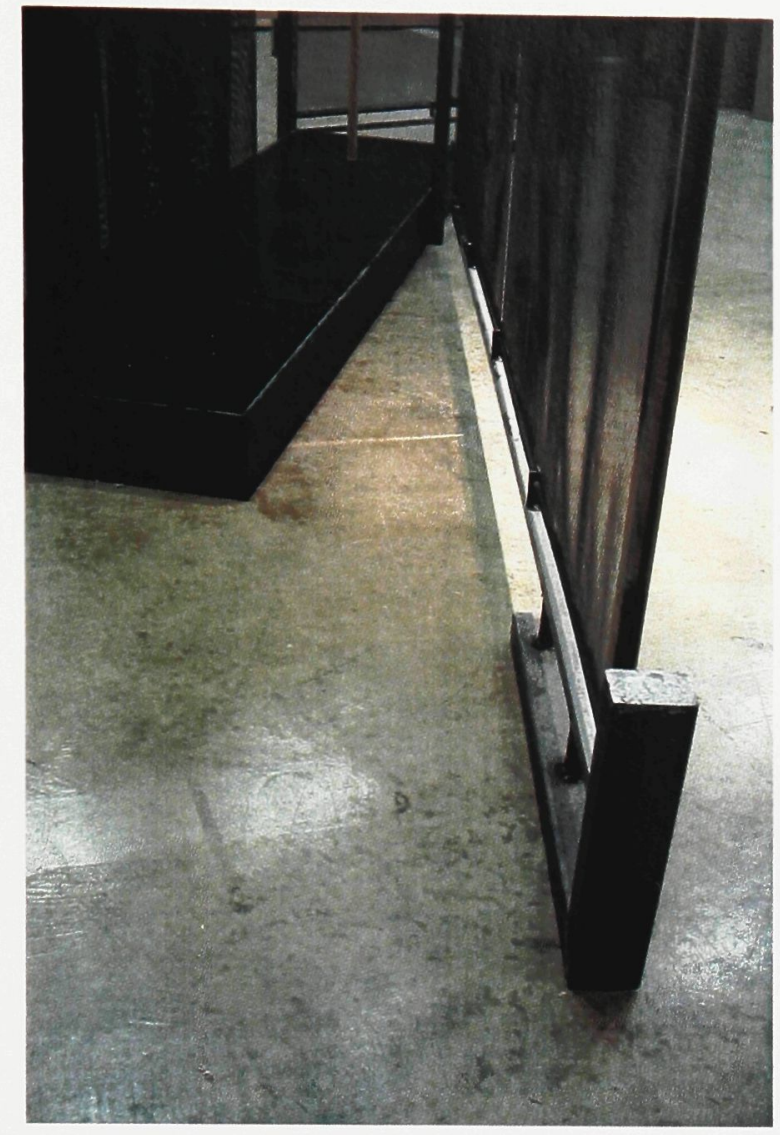

Figure 29
Screen Detail

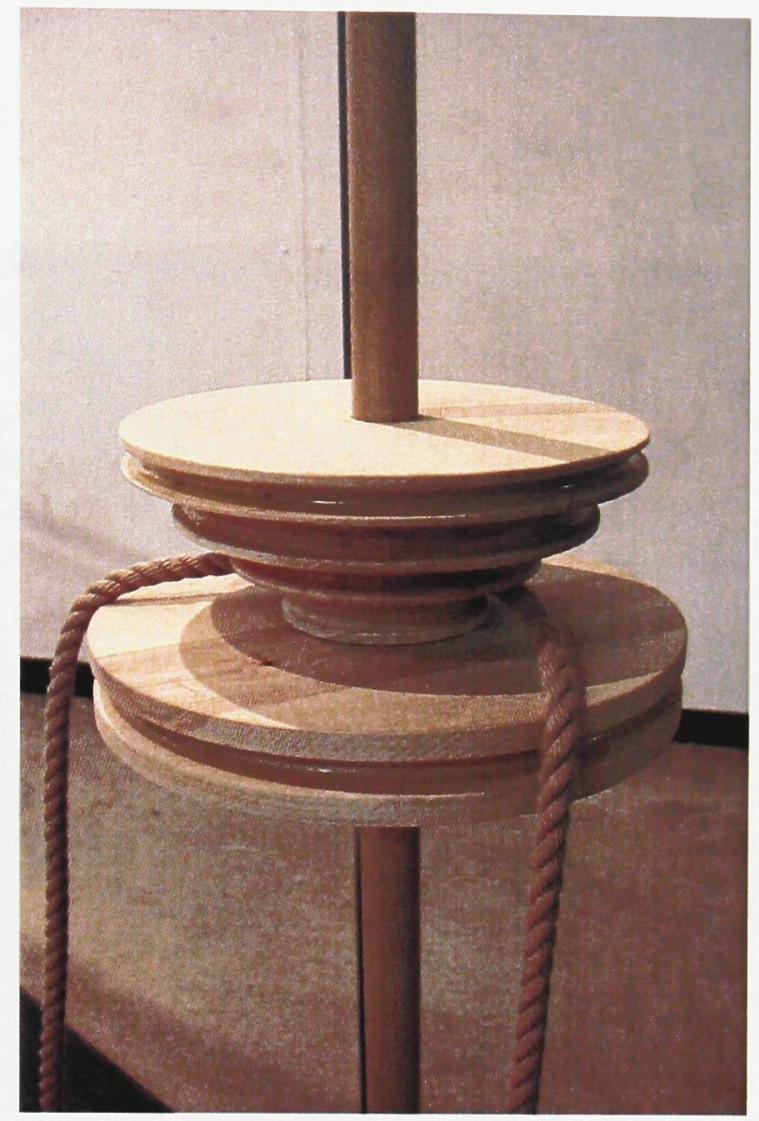

Figure 30

Pulley Detail

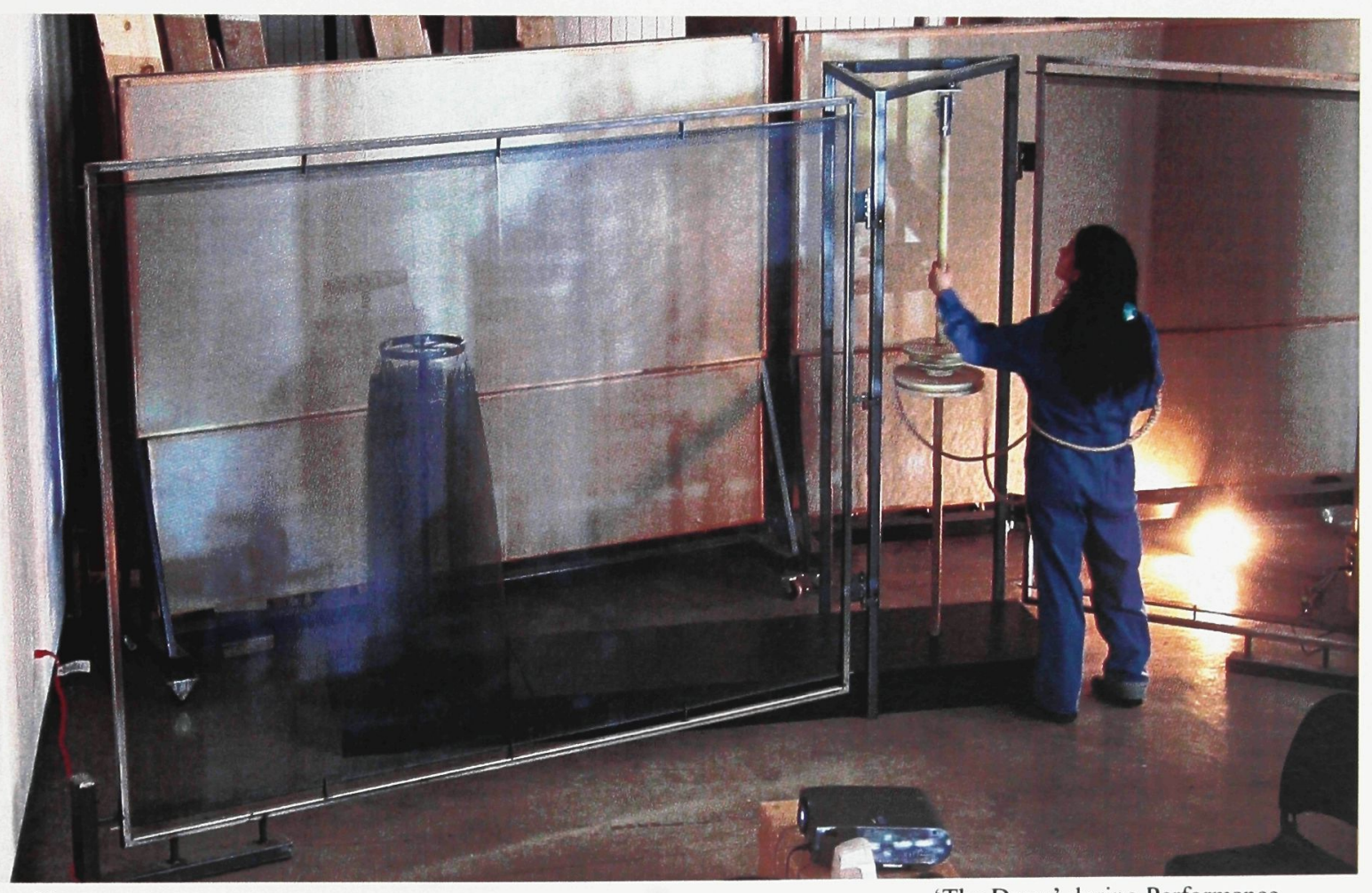

Figure 31

'The Dress' during Performance 


\subsection{Act Four: Drawing From Memory}

A 10 foot rendering was drawn

as a way to expand upon the acts that have already been performed. This drawing was performed as a way to reflect upon previous acts by allowing them to overlap on paper.
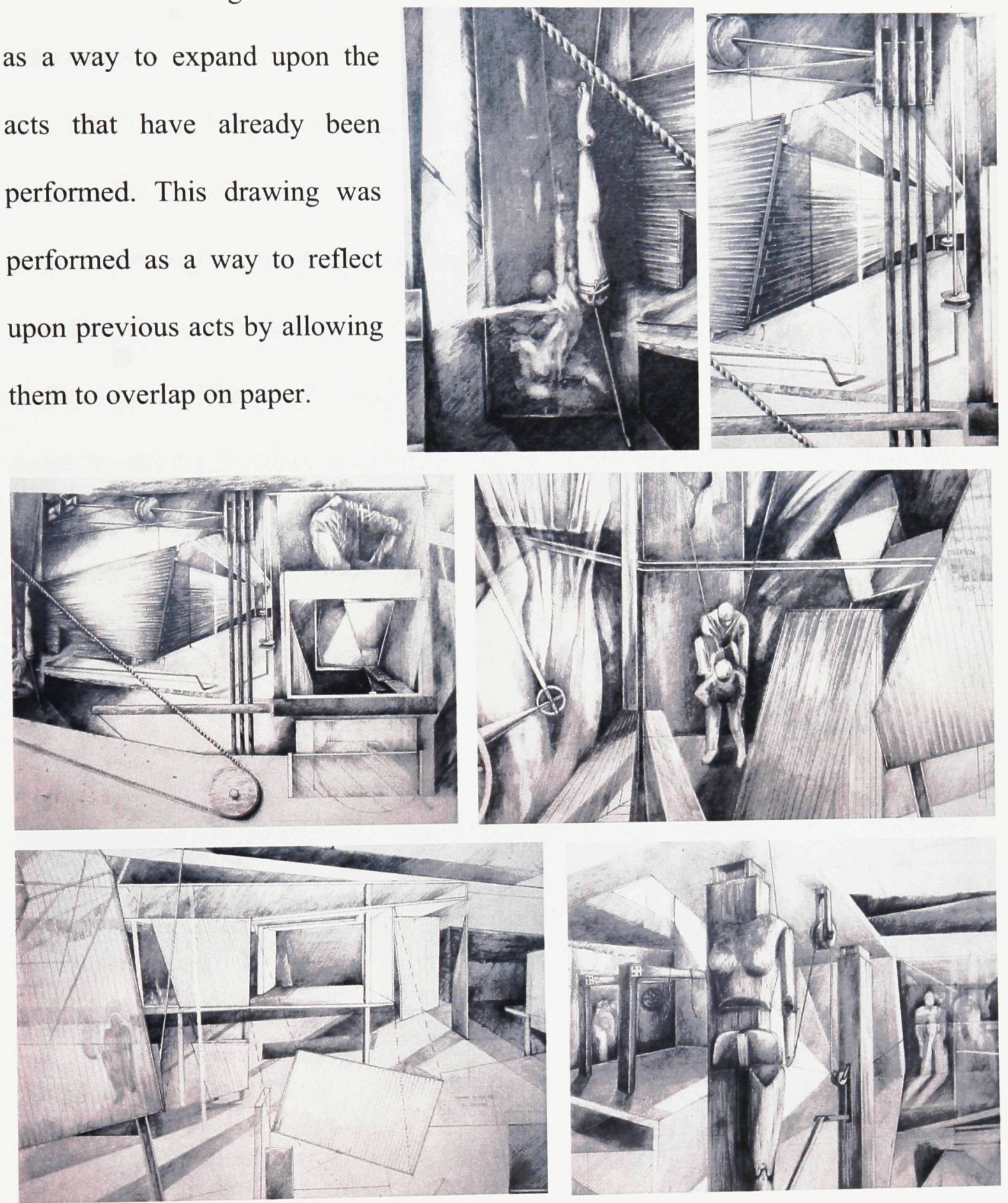

Figure 32 Drawing Details 


\subsection{Act Five: Building the Laboratory}

\section{Intentions}

This building is founded upon the premise that form can incite a kind of temporal folding that interrupts the habitual consciousness of a pedestrian or an inhabitant so as to prolong the moment of exchange between subject and object. Instead of assuming that the building will engender a specific kind of experience, the intent is that the building will delay the moment of encounter between subject a passerby and the building itself, long enough to provoke self-conscious reflection.

\section{Site}

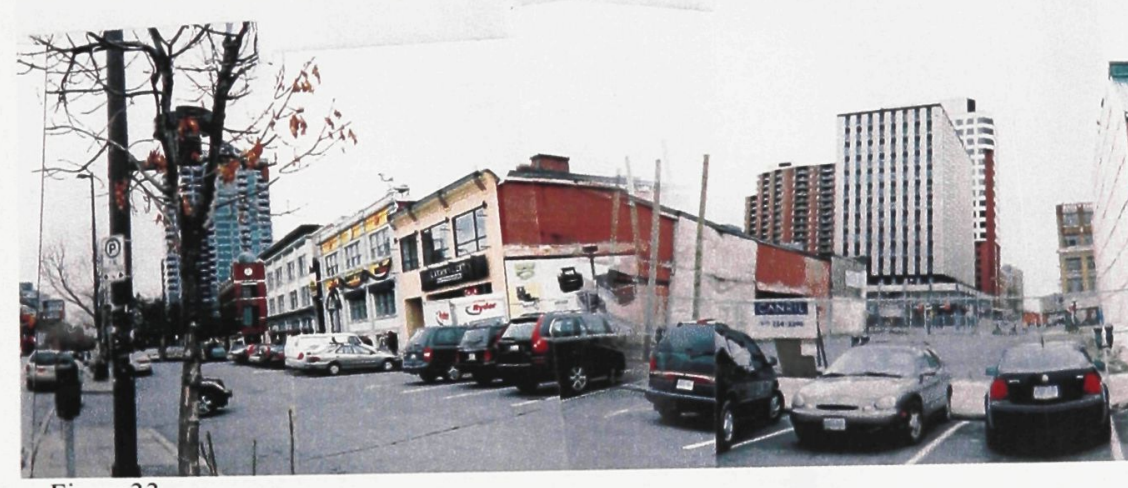

Figure33

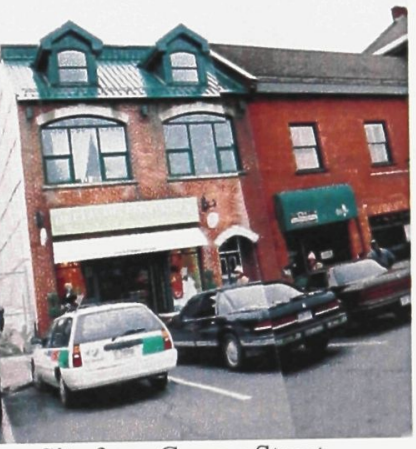

Site from George Street

The building is to be situated on a site that bridges George Street and Rideau Street in the Market area of downtown Ottawa. The scale of the neighborhood provides the opportunity for many layers of engagement. Not only is this a very Pedestrian oriented neighborhood, but the By-ward market to the north of the site ensures that pedestrians will not only be walking alongside the site, but will walk 
towards it to some degree. Threfore, the façade of any proposed building can be engaged with at both a close range, and also from afar. The south end of the site is on Rideau Street., which bears both a high volume of pedestrian traffic as well as vehicular traffic. Additionally, the site will also be looked down upon from higher vantage points, because of the many office and condominium towers dispersed throughout the neighborhood. Additionally, the site is in close proximity to the existing Dance Lab, the Ottawa Art Gallery, the Saw Gallery and the Ottawa School of Art. Furthermore, the National Gallery and the National

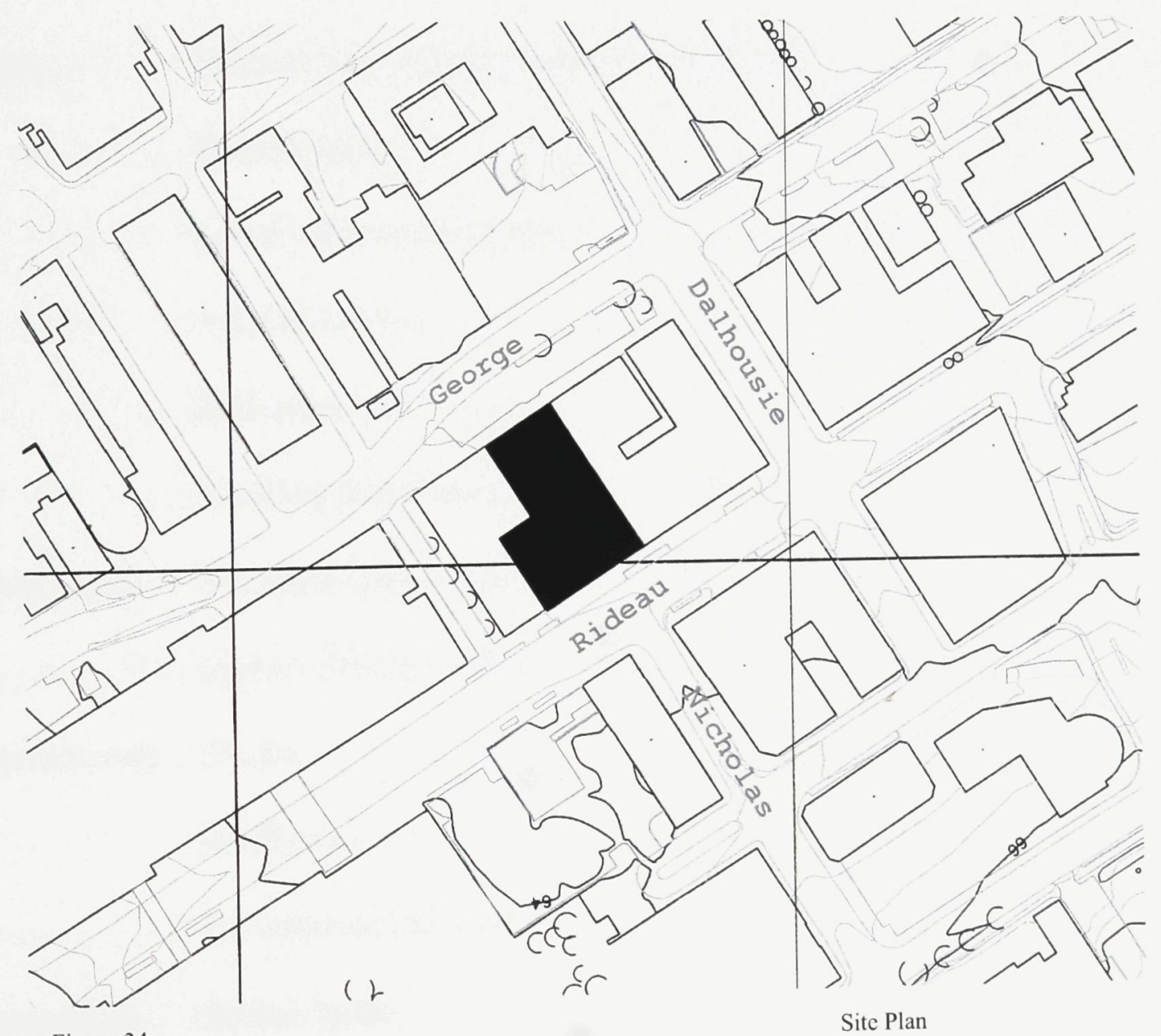

Figure 34 
Arts Centre are both in close proximity to this site. As an interdisciplinary laboratory, it is advantageous for these institutions to be in close proximity, in order to create relationships.

\section{Programme}

The building programme is modeled after the dance lab performance, and incorporates five disciplines into one experimental theatre building: dance, architecture, sound, video and light labs.

Main Theatre (seat capacity approximately 220)

Theatre lobby

Library/Resource Centre

Administration

Rehearsal hall

Archival and Storage space

Dance Lab Mirrored Dance Studio

Dancer change rooms

Architecture Studio

Workshop

1:1 construction space

Sound Lab Sound Studio

Editing Room 


\section{Storage Space for Equipment}

\section{Light Lab Light Studio}

Dark Room

Storage Space for Lights

Video Lab Video sound stage

Editing Room

Projection room

As an experimental theatre, this project is not geared towards the final production as a full and finished product, but rather at exploring the creative act through the medium of theatre. In addition to furthering the understanding of each individual discipline within itself, the building brings all five disciplines together to engage in ongoing dialogue. Hence the studios are not intended for permanent residents from each of the five disciplines, rather, the residents are always changing, so different members of each discipline can come together to engage in collaboration. In this way, the characters of the disciplines themselves are always changing, thereby contributing to a unique product.

\section{The interdisciplinary laboratory}

When approaching the building's George Street façade, one sees oversize double doors spanning almost three storeys in height. This operable façade is constructed out of translucent polycarbonate that is opaque during the day. At night they 
become massive lanterns of light and shadow that transform as the lights behind them change. If it is warm enough, these doors can remain open. The façade doors are controlled by a giant gearbox located halfway between first and second storey to the left of the doors. To operate the controls, one must enter the gear box to turn a two meter wide wheel.

Beginning with the operable George Street façade, the main core of the building is articulated as a kind of exploded corten steel box that moves from the lobby to the theatre proper, to the projection surface, the dance studio, and back out to the Rideau street façade. If the façade is open, the lobby can also be used as a stage for a street performance. This is accommodated by a lighting grid above and a projection booth that faces both the façade and the lobby. The corten steel box is contained within a concrete shell that is continuous from floor to wall to ceiling, opening up towards Rideau Street. On the main floor, this surface defines a working platform upon which the theatre sits. Flexible undulating walls allow it to open up in order to accommodate set building, and changing requirements of the theatre. The light, sound, video, architecture, and dance labs are all immediately adjacent to this theatre. In effect, the theatre itself is the central convergence point of the building. All laboratories open onto the theatre to accommodate the collaborative efforts of the different disciplines. The light, sound and video labs are directly east of the theatre. The architecture lab is directly west of the theatre, in a sunken construction pit. Also contained within the building is a leasable space that can accommodate a restaurant or café, as well 
as small walk-up residential units that can either house the transient interns of the lab, or can be rented out individually as apartment units. Both the leasable space and the apartments are located to the west of the main theatre core. The main entrance of the building is common to both residents of the apartments as well as residents of the theatre.

Proceeding south beyond the theatre is a large projection surface that is continuous for the full height of the theatre. On the other side of this screen on the second storey mirrored dance studio this studio looks onto Rideau street. The mirrored roof of this dance studio lifts off of the walls to reflect street activity. Like the George Street façade, the Rideau street façade is operable from a gear box. But instead of opening literally onto the street, this façade changes from the second storey. Essentially, the façade directly under the ceiling mirror is broken into fragments that can be moved closer together, or further apart. The distance between these fragments would change the transparency of the façade. These glowing doors of the George street façade are raised above street level, where the window screens can open to provide visual porosity, acting as a lantern to illuminate Rideau Street. 


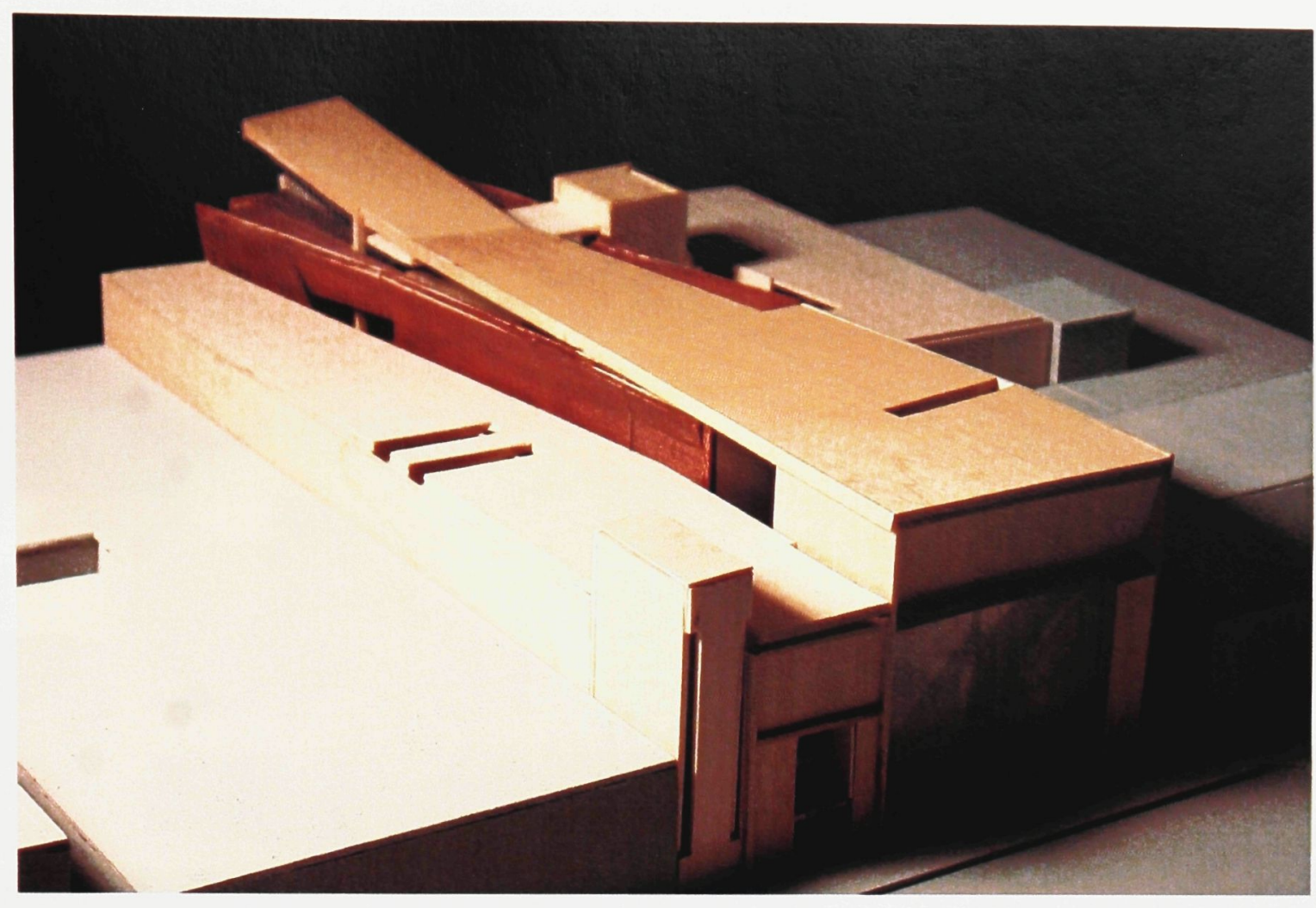

Figure 35

Roof View from George Street

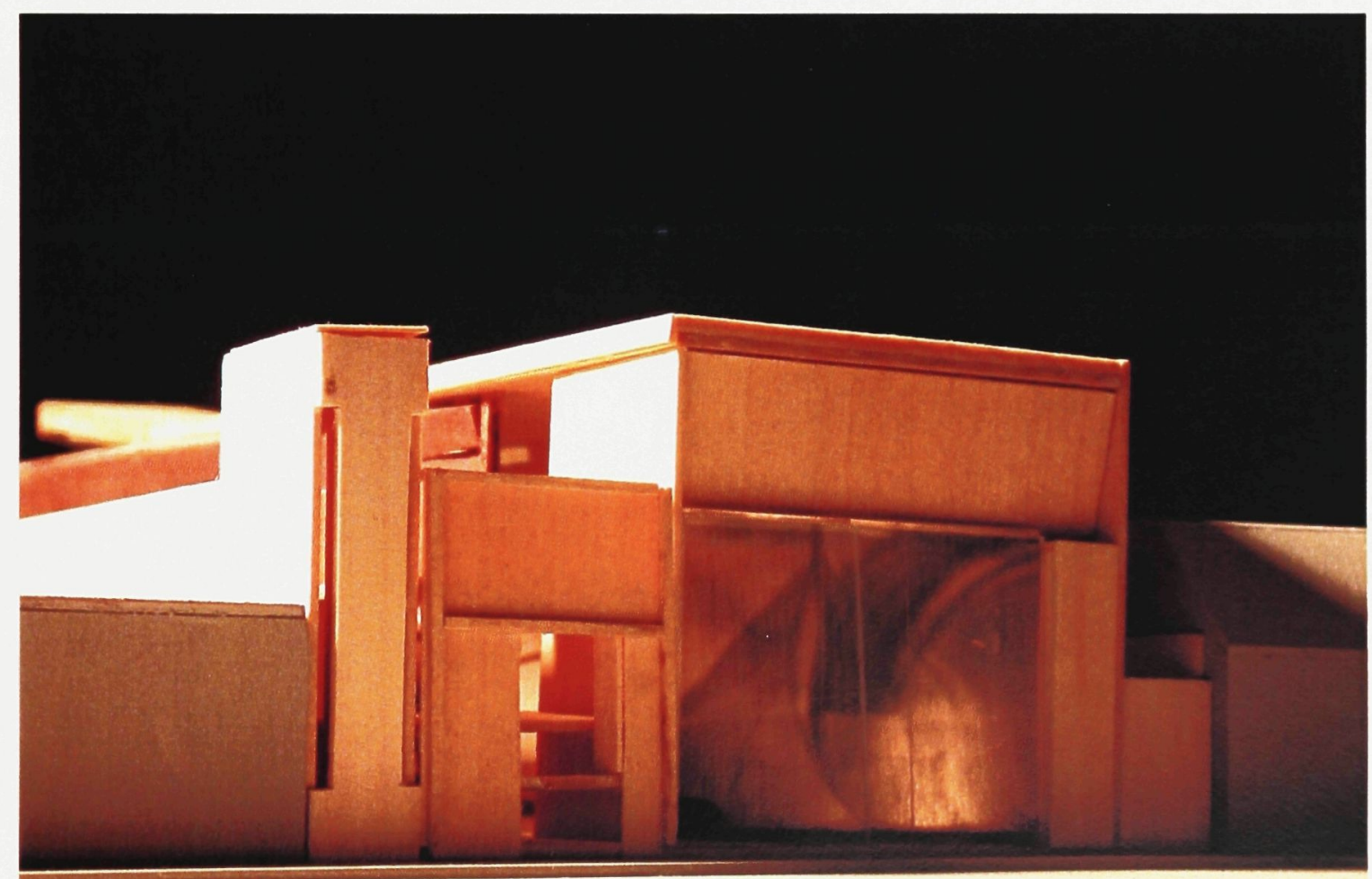

Figure 36

George Street Facade 

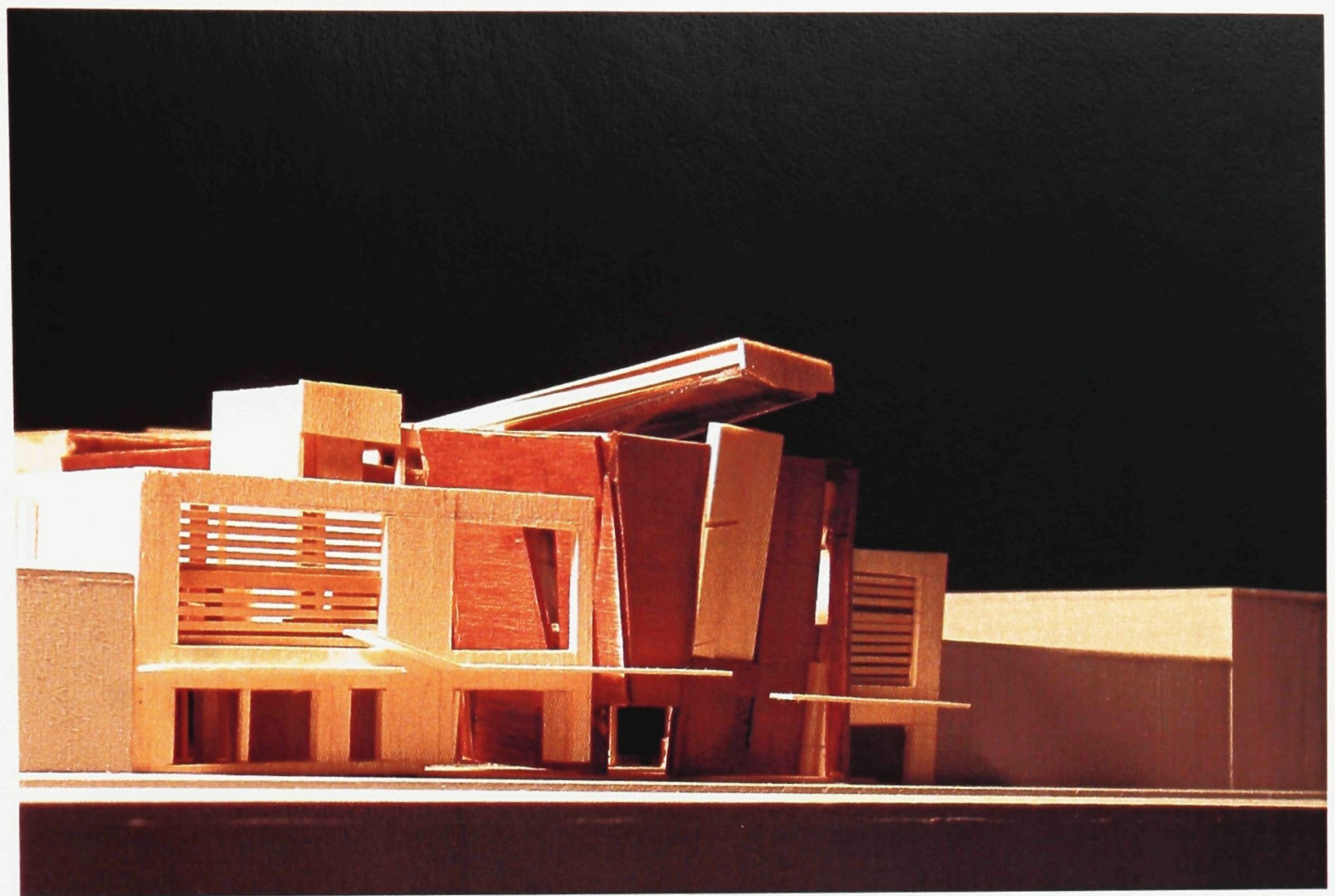

Figure 37

Rideau Street Facade

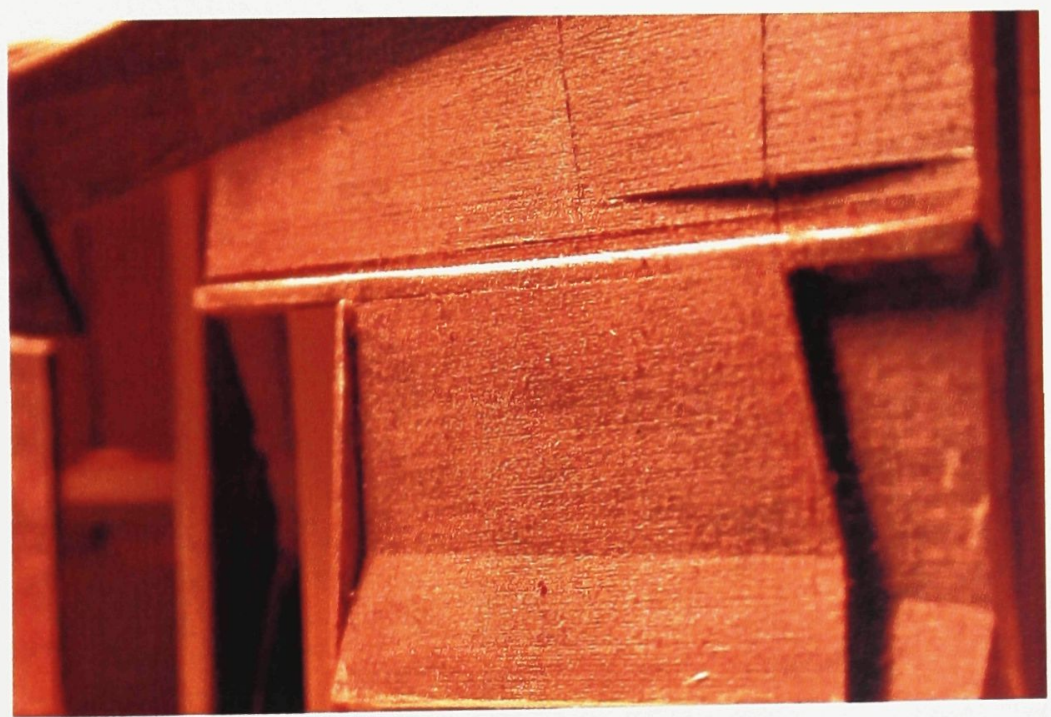

Figure 38
Theatre Detail

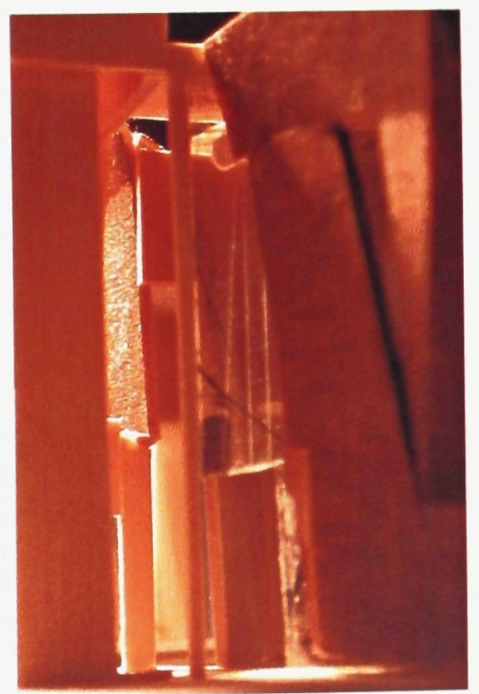

Figure 39 Main Entrance 

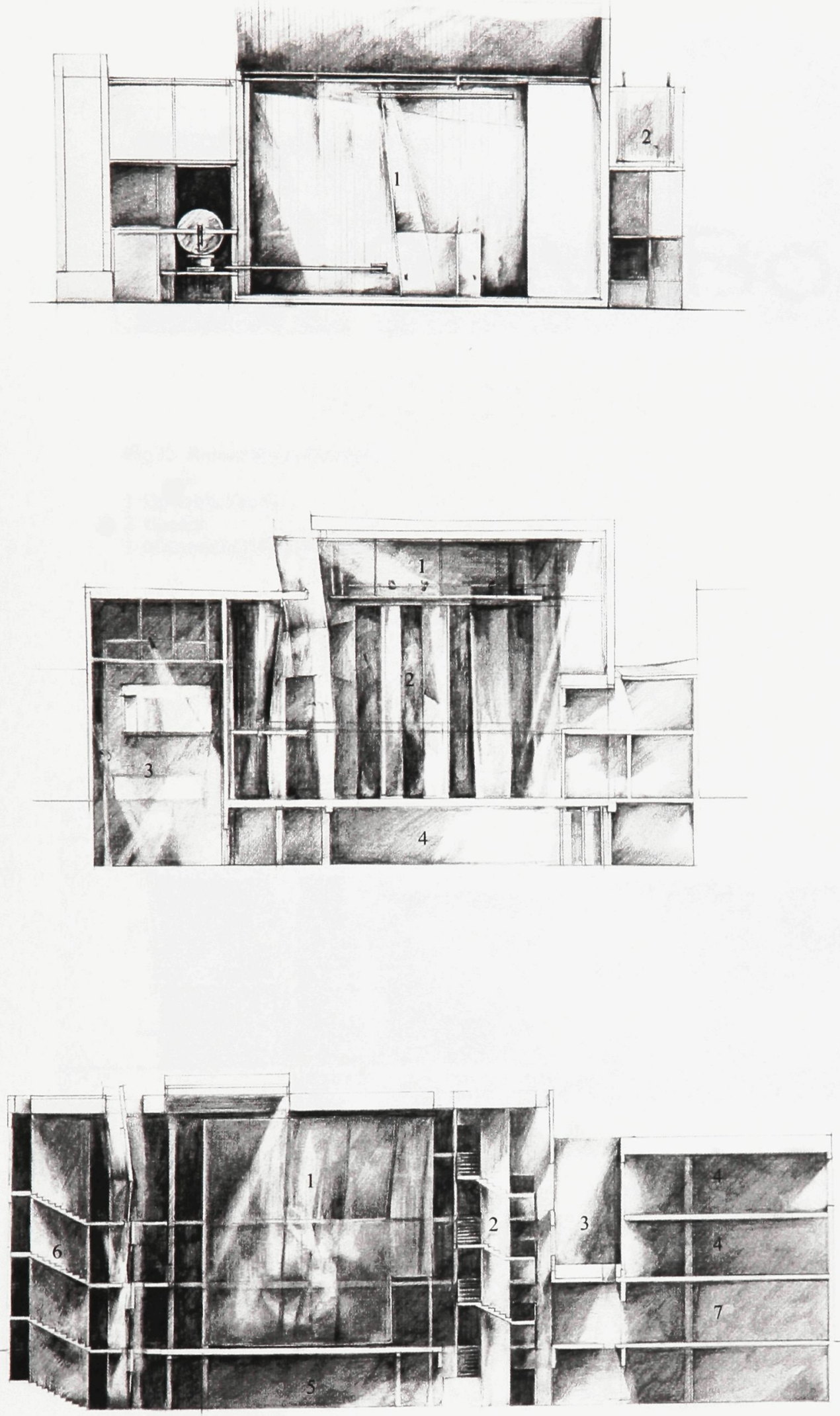

Fig. 40 George Street Elevation

1 Operable Façade

2 Projection Screen

Fig. 41 Cross Section Through Theatre

1 Theatre

2 Projection Screen Beyond

3 Light Laboratory

4 Atelier

Fig. 42 Cross Section in Front of Scre

1 Projection Screen

2 Main Stair

3 Garden

4 Apartments

5 Atelier

6 Theatre Stair

7 Leasable space 


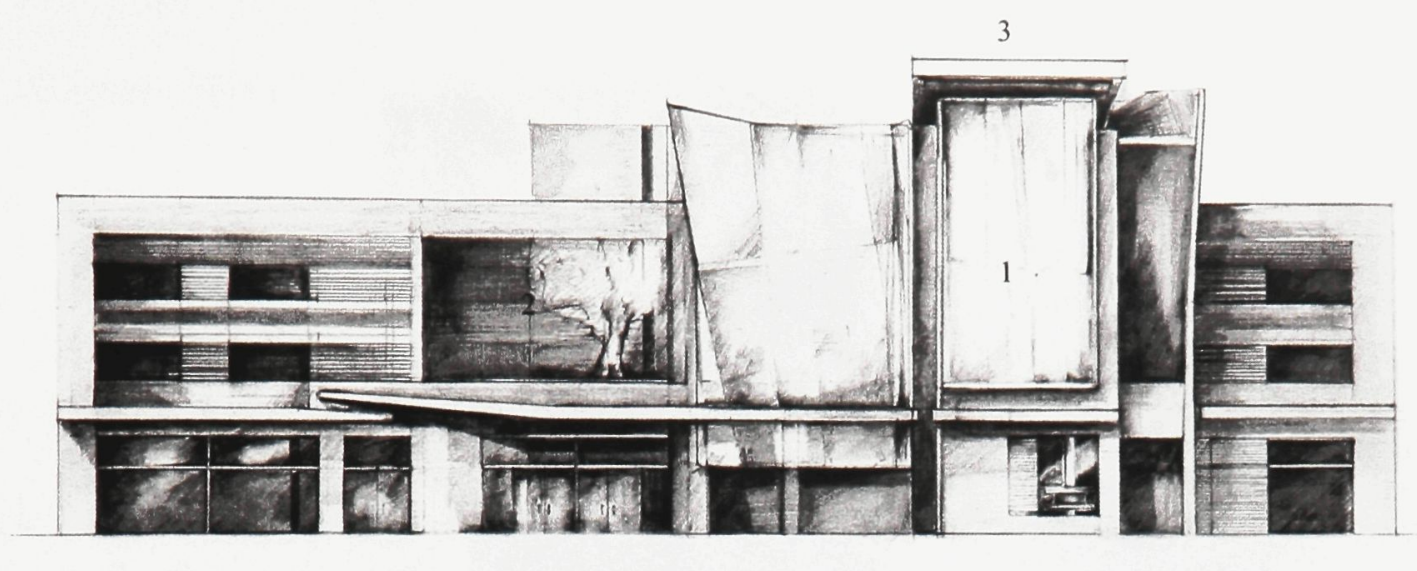

Fig 43 Rideau Street Elevation

1 Operable Façade

2 Garden

3 Mirrrored Ceiling (Below)

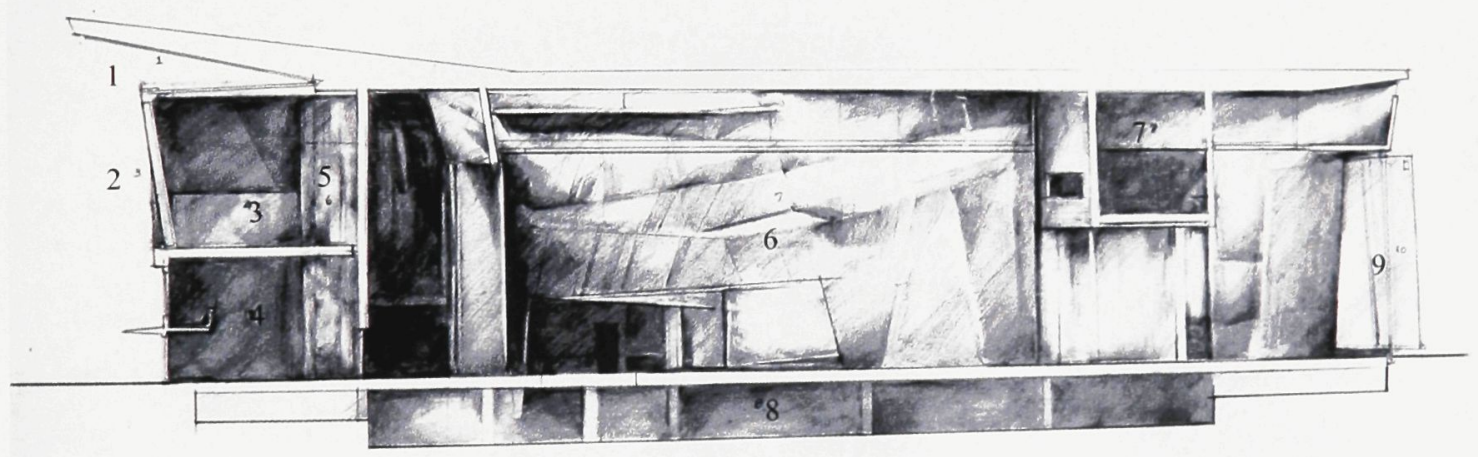

Fig 44 Longitudinal Section
1 Mirrored Ceiling
2 Operable Façade
3 Dance Studio
4 Resource Centre
5 Projection Screen
6 Theatre
7 Projection Booth
8 Atelier
9 Operable Façade/Theatre Entrance 


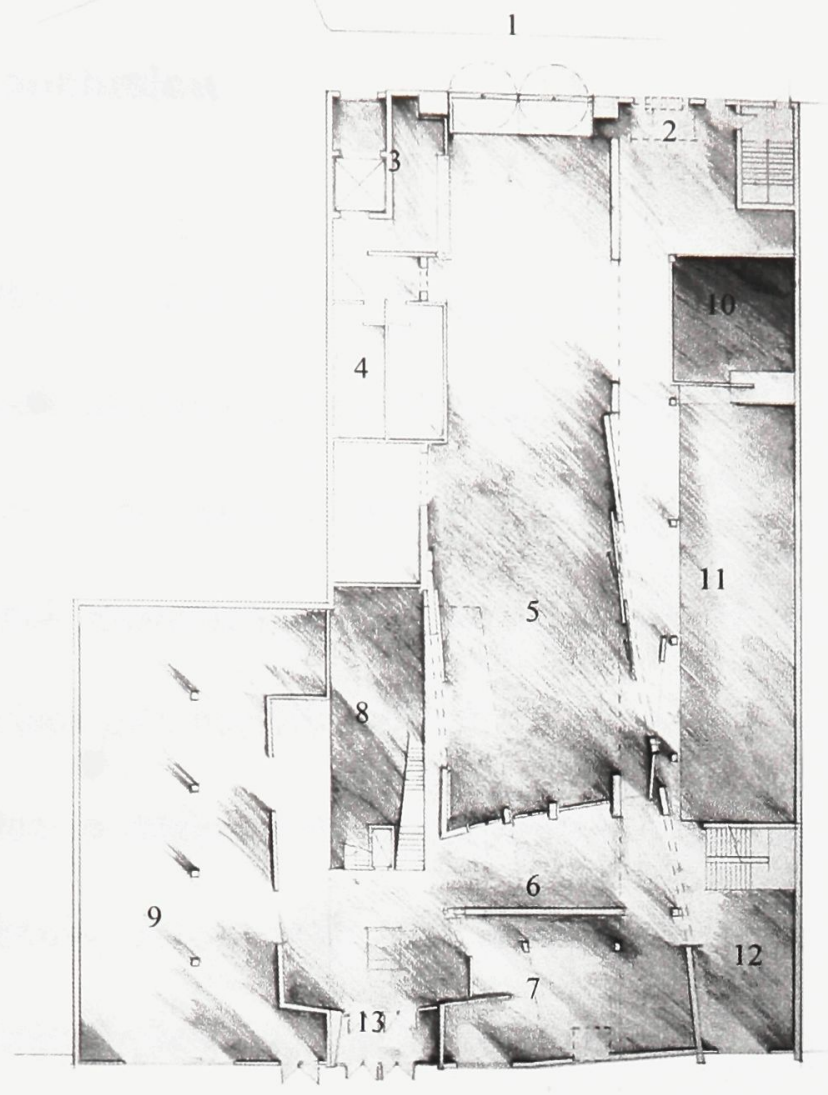

Fig. 45 Main Floor Plan

1 Operable Façade/Theatre Entrance

2 Façade Controls (above)

3 Coat Check

4 Washrooms

5 Theatre Space

6 Projection Screen

7 Resource Centre

8 Large Scale Construction Pit

9 Leasable Space

10 Light Laboratory

11 Studio Space

12 Administration

13 Main Entrance

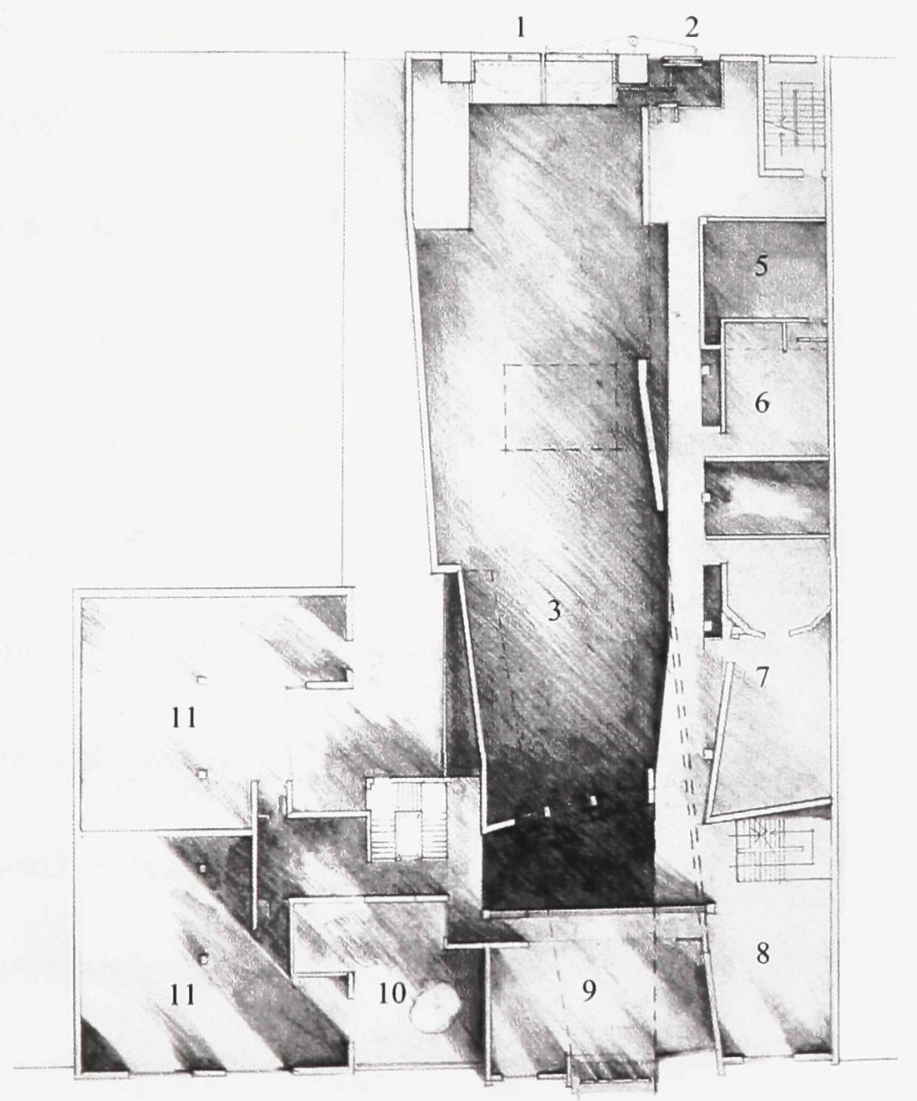

Fig.46 Second Floor Plan

1 Operable Façade

2 Façade Control

3 Theatre Below

4 Projection Screen

5 Light Laboratory

6 Dark Room

7 Recording Studio

8 Video Editing Lab

9 Dance Studio

10 Garden

11 Apartment 


\section{Conclusion}

In "Weak Architecture," Ignasi de Sola-Morales contends that the disappearance of any universal point of reference has resulted in a situation where every architect must posit his or her own goal and grounding. ${ }^{58}$ Attempts to resurrect a rational epistemology have led us to our contemporary situation, defined by its very lack of a universal system of organization. "Weak Architecture" is an essay written in response to this groundless state that resigns itself to a condition of weakness, recognizing that architectural theory can only be a weak link to facilitate reflection upon built form, not the means to an end. The response outlined in "Weak Architecture" is not a formal strategy for creating architecture that is weak, but is instead a discussion that empowers the reader with the authority to interpret. Ultimately, weak architecture is the notion that human reconciliation with the material world can only occur within the temporal realm through the medium of the human body.

This kind of corporal reconciliation with life is central to Henri Bergson's ideas on the human condition. For Bergson, the only absolute in the world is found in duration, the unceasing creative force of life that occurs only within the temporal realm. Our inability to understand duration is the result of our own flawed intellect. To Bergson, the only means of reconciling life with the understanding of life is through a self-conscious intuition, an intellectual 
awareness of our own bodies in temporality. In Bergson's model, the shape of this visceral awareness is contingent upon the personal memory, which is not merely an objective recording of the events of life, but rather the factor that renders our experiences malleable and expansive.

This project employs the self-reflective encounter as the basis for architectural exploration, spurred by an interruption of the habitual in the unforeseen place and time. The work uses a temporal act of creation in the form of a collaborative dance as the basis for further creative exploration. Through a process of self-reflection and creative action, the initial event expands upon itself to arrive at the product.

\footnotetext{
${ }^{58}$ Sola-Morales, "Weak Architecture, 59.
} 


\section{List of Illustrations}

1. Gordon Matta-Clark, Circus or the Caribbean Orange, 1978, in Pamela M. Lee, Object to be Destroyed (Cambridge and London: The Mit Press, 2001) 159 .

2. Gordon Matta-Clark, Circus or The Caribbean Orange, 1978, in Lee, Object to be Destroyed, 155.

3. Gordon Matta-Clark, Circus or The Caribbean Orange, in Lee, Object to be Destroyed, 157.

4. Vernon March, Canada's National War Memorial, 1939, in War Memorials Aug. 2005 http://www.rockies.net/ spirit/images/natwarmem.gif

5. Peter Eisenman, Holocaust Memorial, model, in The Design Boom 26Aug $2005<\mathrm{http}: / / \mathrm{www} \cdot$ designboom.com/eng/interview/eisenman.html>

6. Peter Eisenman, Holocaust Memorial, Model, Aug. 2005 $<$ http://bss.sfsu.edu/jacksonc/H317/Holocaust\%20Memorials/eisenmann.j $\mathrm{pg}>$

7. Dance Steps installed in main street of the School of Architecture

8. Basic left turn, Tango, Oct. $2004<$ http://www.umich.edu/ umbdc/DanceSyllabus/haagr/am_tango/am_tango _bronze/amtg_leftturn_lady.htm> 
9. Basic left turn, Tango, Oct. $2004<$

http://www.umich.edu/ umbdc/DanceSyllabus/haagr/am_tango/am_tango _bronze/amtg_leftturn_man.htm>

10. Still sequences from recorded footage of the dance steps

11. Dance Steps

12. Detail of Dance Steps

13. Detail of the story board

14. Week One: Shauna Elton at work with the dancers

15. Week two: Dancers rehearsing a sequence

16. Week two: Dancers climb on Koichi Yano

17. Koichi Yano

18. group wall sequence

19. Marie-Claire forte is lifted

20. Cecily Greenfield and Marie-Claire Forte perform the final sequence

21. Sketch of the Walter and Amy Dance Machine

22. Walter and Amy in source performance for the dance for the construction one

23. Construction One: 'Walter and Amy' Machine

24. Construction One: Pulley System

25. Construction One: Support detail

26. "Walter and Amy' machine in operation

27. Cecily's solo 
28. Construction Two: The Dress Spinning Machine

29. Construction Two: Screen Detail

30. Construction Two: Pulley Detail

31. Construction Two in Operation

32. Drawing Details

33. Photograph of Site from George Street

34. Site Plan

35. Roof View From George Street

36. George Street Facade

37. Rideau Street Facade

38. Theatre Detail

39. View From Main Entrance

40. George Street Elevation

41. Cross Section Through Theatre

42. Cross Section in Front of Screen

43. Rideau Street Elevation

44. Longitudinal Section

45. Main Floor Plan

46. Second Floor Plan 


\section{Bibliography}

Beaune, Jean-Claude. "The Classical Age of Automata: An Impressionistic

Survey from the Sizteenth to the nineteenth Century," Fragments for a History of the Human Body Part One, ed. Michel Feher et al. New York: Zone Books, 1989.

Bergson, Henri. An Introduction to Metaphysics. Trans. T.E. Hulme. Indianapolis and New York: The Library of Liberal Arts, 1955.

---. Matter and Memory. Trans. N.M.paul and W.S.Palmer, New York: Zone Books, 1991.

---. Creative Evolution. Trans. Arthur Mitchell. New York: the Modern Library, 1944.

Deleuze, Gilles. Bergsonism. Trans. Hugh Tomlinson and Barbara habberiam. New York: Zone Books, 1991.

Grosz, Elizabeth. Architecture from the Outside. Cambridge: The MIT Press, 2001.

Lee, Pamela M.. Object to be Destroyed. Cambridge and London: the MIT Press, 2001.

Kockelkoren, Petran. Technology: Art, Fairground and Theatre. Rotterdam: NAi Publishers, 2003.

Perez-Gomez, Alberto. Architecture and the Crisis of Modern Science. Cambridge and London: The MIT Press, 1996.

Sola-Morales, Ignasi de. "Weak Architecture," Differences: Topographies of Contemporary Architecture. Trans. Thomson, Graham. Cambridge: The MIT Press, 1999. 57-71.

Pierce, James Smith. From Abacus to Zeus. New Jersey: Prentice Hall, 1998.

Ursprung, Philip, ed. Herzog and de Meuron: Natural History. Montreal: Canadian Centre for Architecture and Lars Muller Publishers, 2002. 
Vattimo, Gianni. The End of Modernity: Nihilism and Hermeneutics in Postmodern culture. Trans. Jon R. Snyder. Baltimore: The Jon Hopkins University Press, 1991.

Von Kleist, Heinrich. "On the Marionette Theatre." Fragments for a History of the Human Body Part One, Ed. Michel Feher et al. New York: Zone Books, 1989. 